\title{
Vehicle routing with private fleet, multiple common carriers offering volume discounts, and rental options
}

\author{
Christian Gahm ${ }^{\mathrm{a}, *}$, Christian Brabänder ${ }^{\mathrm{b}}$, Axel Tuma ${ }^{\mathrm{a}}$ \\ ${ }^{a}$ Chair of Business Administration, Production E Supply Chain Management, Augsburg University, D-86135 Augsburg, Germany \\ ${ }^{\mathrm{b}}$ Chair of Business Administration, Controlling and Logistics, University of Regensburg, D-93053 Regensburg, Germany
}

\section{Keywords:}

Vehicle routing

Private vehicles

Common carrier

Rental options

Volume discounts

Variable neighborhood search

\begin{abstract}
A B S T R A C T
The problem addressed in this paper extends the vehicle routing problem with private fleet and common carriers by three aspects: two types of rental options, a cost function considering volumes and distances, and volume discounts offered by the common carriers. For its solution, we present a mixed integer program and three heuristics based on Variable Neighborhood Search. The computational analysis demonstrates the suitability of these heuristics and the positive effects of two newly introduced mechanisms. Analyzing the interdependencies between available outsourcing options and economic benefits, it shows that a subset of options is sufficient to reduce costs remarkable.
\end{abstract}

\section{Introduction}

In this article, a comprehensive extension to the (capacitated) vehicle routing problem with private fleet and common carriers (VRPPC) is presented. The VRPPC, as discussed over the last ten years, tackles the problem of delivering products from a single central depot (e.g., shipping company) to customer locations. This task is accomplished either by the company's privately owned homogeneous or heterogeneous vehicle fleet (self-fulfillment) or by employing external common carriers (subcontracting), i.e., less than truckload (LTL) carriers. This standard VRPPC consists of a selection decision combined with a clustering decision and a routing decision. The first decision is to select one of the two delivery modes for each customer to be served; the second decision comprises the standard vehicle routing problem for the private fleet serving the assigned customers. Concerning the VRPPC, each customer must be served by exactly one vehicle of the limited private fleet or by exactly one external carrier (no split-delivery), every route of the private vehicles start and end at the depot, and vehicles of the private fleet have a specific capacity and perform at most one route per day. The objective is to minimize total delivery costs to serve all customers. Regarding the two delivery modes self-fulfillment and subcontracting, it is assumed that full truckload (FTL) deliveries executed by own vehicles are always cheaper than other delivery modes. Nevertheless, great saving opportunities are in subcontracting LTL deliveries to external carriers.

In this paper, we extend the VRPPC by three aspects. First, we consider two exclusive rental options as additional options for subcontracting: one with a rental fee charged on a route basis (mileage) and the second with a rental fee charged on a daily basis (see e.g., Krajewska and Kopfer, 2009). Both rental options provide the opportunity to reduce total delivery costs

\footnotetext{
* Corresponding author.

E-mail addresses: christian.gahm@wiwi.uni-augsburg.de (C. Gahm), christian.brabaender@wiwi.uni-regensburg.de (C. Brabänder), axel.tuma@wiwi. uni-augsburg.de (A. Tuma).
} 
by increasing the FTL delivery volume. The latter rental option is identical to the consideration of (full) truckload carriers that account for fixed cost per load up to a given capacity (see e.g., Rieksts and Ventura, 2008 or Toptal and Bingöl, 2011). Second, a more realistic concave freight function based on volumes and distances is integrated to determine the costs of LTL carriers (see e.g., Krajewska and Kopfer, 2009). The third aspect is the consideration of volume discounts offered by (some) LTL carriers. Generally, volume discounts are a financial incentive used to foster demand and can be characterized as all-unit quantity discounts or incremental quantity discounts (Wilcox et al., 1987; Weng, 1995). From the view of a common carrier, these discounts provide potential to realize scale effects by freight consolidation in the short term (especially on the inbound route to a distribution center - see Nguyen et al., 2014; Campbell, 1990, on the effects of freight consolidation). In this context, Nguyen et al. (2014) discuss the increase of a customer's order size fostered by volume discounts offered to consolidate delivery orders. Discounts could also be used by LTL carriers to achieve a higher customer density, which leads to decrease in total delivery costs (see, Sun et al., 2015 on this relation). Therefore, the external carrier's operational costs per unit are likely to decrease and these savings could partially be passed to the shipper (to achieve a competitive advantage). Consequently, the shipper's delivery costs will also decrease. This and the previous aspect leads to a heterogeneous set of common carriers defining individually parametrized cost functions. Therefore, the new freight function for common carriers needs to be concave to represent decreasing freight rates depending on volumes and distances and needs to enable carrier-dependent discounts.

Altogether, these aspects lead to a new delivery planning approach for shipping companies addressed in this manner for the first time. We name this new approach the vehicle routing problem with private fleet, multiple common carriers offering volume discounts, and rental options (VRPPCdR). In order to solve problem instances of virtually any size, new and enhanced solution methods based on the principles of Variable Neighborhood Search (VNS) are proposed herein. First enhancement is an explicit shaking mechanism for solution perturbation to support the exploration of the solution space. Second, a distance proportionate selection mechanism is introduced in order to increase the efficiency of the local search procedures.

The structure of the paper is as follows. The literature review in Section 2 examines similar problems in detail and shows the relevance of the new planning approach. The VRPPCdR is formally described in Section 3 and a mixed integer program (MIP) is presented there. The different solution methods and the introduced enhancements are described in Section 4 . The computational analysis in Section 5 shows that the solution methods are suitable to solve the VRPPC and particularly the VRPPCdR. The analysis also shows that the new planning approach is able to reduce delivery costs remarkable and provides managerial insights on the effects of different subcontracting scenarios. Finally, conclusions and potential further research topics are described in Section 6.

\section{Literature review}

Basically, two main research streams address operational transportation planning problems with subcontracting: one investigates the planning problem from the perspective of a freight forwarding company; the other one investigates the perspective of a shipping company. The main difference between both streams lies in the basic planning problem: freight forwarding companies have to solve a pickup and delivery problem (PDP), whereas shipping companies owning a private fleet have to solve a capacitated vehicle routing problem (VRP). Of course, several variants of each of these basic problems are addressed in the literature. Another difference between a shipper and a forwarder is in the objective: While shippers aim to lower their total delivery costs, freight forwarders aim to acquire high volumes in order to generate revenues and lower costs per unit by consolidated full truck loads.

Since this paper takes the shipper's perspective, only a brief review of the relevant literature concerning the freight forwarding perspective is given by selected papers. The paper of Krajewska and Kopfer (2009) is one of the first that combines several subcontracting options in an integrated manner. The authors enhance the underlying pickup and delivery problem with time windows (PDPTW) by external carriers and the two exclusive rental options, described above. The authors called this problem the Integrated Transportation Planning Problem (ITPP; later also called Integrated Operational Transportation Planning - IOTP) and propose a tabu search heuristic extended by special types of moves for the different subcontracting options. In a similar context, Liu et al. (2010) address a task selection and routing problem in collaborative truckload transportation and solve the problem by a memetic algorithm. In contrast to the VRPPC, the authors include external delivery task during the shippers' distribution planning and the private fleet is of unlimited size. Based on the ITPP, Wang and Kopfer (2014) and Wang et al. (2014) formulate the Collaborative Transportation Planning (CTP) problem. Generally, collaborative planning can be seen as a joint decision making process and CTP aims at the reallocation of requests among the partners in a horizontal cooperation. Accordingly, the main difference between the CTP and the ITPP is that CTP bases on an equal partnership, while in ITPP (and also the VRPPC) the players have a hierarchical relationship. Ziebuhr and Kopfer (2014) consider the IOTP from a forwarders perspective extended by compulsory requests, which are only permitted for self-fulfillment or premium subcontracting mode. Therefore, they use a formulation with common carriers and self-fulfillment and apply a large neighborhood search. Defryn et al. (2016) for example also address a vehicle routing problem in a collaborative environment: Based on the selective vehicle routing problem (SVRP), which can be interpreted as a VRPPC, different cost allocation methods for the SVRP in a collaborative environment are analyzed.

The first article that considers external carriers in the perspective of a shipping company originates from Ball et al. (1983). The authors investigate a fleet-size optimization problem, covering the option to outsource destinations to one external car- 
rier. Klincewicz et al. (1990) and Hall and Racer (1995) also aim to determine an optimal fleet size when external carriers are available. When the private vehicle fleet contains only a single vehicle, the problem is similar to Prize Collecting Traveling Salesman Problem (PCTSP) without rewards for visiting nodes. Volgenant and Jonker (1987), Balas (1989), Bienstock et al. (1993), and Diaby and Ramesh (1995) tackled this problem (see also Balas, 2007).

The VRPPC with a heterogeneous private fleet as described in Section 1 is first examined by Chu (2005). Chu (2005) generally assumes that FTL deliveries are always cheaper to be delivered by own vehicles, whereas there might be saving opportunities in subcontracting LTL deliveries to an external carrier. In their paper, the costs of the external carrier are determined by a linear function of the Euclidean distance between the depot and the customer, but are independent of demand quantity. Chu (2005) proposes a 3-step heuristic: selection of customers to be served by the external carrier, initial solution construction by a modified savings algorithm (Clarke and Wright, 1964), and solution improvement by a sequence of intra-route and inter-route exchanges. The Selection, Routing and Improvement heuristic (SRI) proposed by Bolduc et al. (2007) outperforms Chu's heuristic by using two initial solutions and a $\lambda$-interchange procedure as first proposed by Osman (1993). Even better results for the VRPPC instances used by Chu (2005) and Bolduc et al. (2007) are reported in Bolduc et al. (2008). In contrast to Chu (2005) and Bolduc et al. (2007), who account for fixed costs independently of whether a private vehicle is used, Bolduc et al. (2008) account for these private vehicles' fixed costs only if the corresponding vehicle is actually used (the authors also apply a linear function for the costs of the external carrier). Their Randomized construction Improvement-Perturbation heuristic (RIP) combines a descent strategy with two diversification strategies: a randomized savings construction phase and a perturbation mechanism. This RIP-heuristic also performs better (compared with SRI) when applied to instances originating from Christofides and Eilon (1969) and Golden et al. (1998). Côté and Potvin (2009) developed a tabu search heuristic (TS) that achieves better results than does the RIP heuristic when applied to the instances with a homogeneous private vehicle fleet (heterogeneous vehicles are not considered at all). With the extension of this tabu search heuristic by ejection chains (TS+), Potvin and Naud (2011) have further improved the results for homogeneous and heterogeneous private vehicle fleets. However, the computation times of the tabu search heuristics TS and TS+ are significantly greater than are those of the RIP heuristic.

Stenger et al. (2013b) extended the VRPPC by multiple depots and called their problem multi-depot vehicle routing problem with private fleet and common carriers (MDVRPPC). Their Adaptive Variable Neighborhood Search (AVNS) algorithm uses cyclic-exchange neighborhoods and incorporates an adaptive mechanism to bias the random shaking step. The AVNS is also evaluated by selected VRPPC instances (only instances with a homogeneous vehicle fleet are considered) and outperforms the RIP and TS algorithms. Furthermore, AVNS is almost equivalent to TS+ in terms of solution quality but requires significantly less computation time. Vidal et al. (2015) consider three different problems, each a particular variant of the vehicle routing problem with profits and customer selection. The authors propose a new large neighborhood search based on "exhaustive" solutions that are embedded within three heuristic frameworks (MS-LI, MS-ILS, and UGHS). Regarding the VRPPC, UGHS slightly outperforms AVNS in terms of solution quality but requires more than twice as much computation time.

All these papers focusing on the VRPPC consider solely the subcontracting to external LTL carriers. Only Kopfer and Wang (2009) consider all three subcontracting options. The authors extend the VRP to the Vehicle Routing and Forwarding Problem (VRFP) and their short evaluation based on very small test instances solved by the commercial solver CPLEX shows the economic benefit of sub-contracting in general.

There are several papers that consider volume discounts when planning the inbound transportation from suppliers (see e.g., Tersine and Barman, 1991; Sheen and Tsao, 2007; Tsao and Lu, 2012). To the best of our knowledge, only Stenger et al. (2013a) consider volume discounts in the outbound transportation (distribution) planning, when discussing routing with subcontracting. Here, Stenger et al. (2013a) extend the MDVRPPC of Stenger et al. (2013b) by quantity discounts and a minimum demand to be delivered by the private fleet. They call it the Prize-Collecting Multi-Depot Vehicle Routing Problem with Non-Linear costs (PCMDVRPNL) and propose a linear cost function and a non-linear stepwise cost function that depends on the vehicle capacity. This capacity is assumed to be identical for all vehicles of the private fleet and also for the vehicles of the LTL carrier. Both cost functions are linked with a maximal discount factor. Similar to Stenger et al. (2013a), we use a decreasing freight rate with a minimum freight rate but in contrast to them, we consider heterogeneous vehicles and heterogeneous common carriers having cost functions with individual parameters (e.g., discount factors).

Summarizing the literature review, there is no planning approach that simultaneously considers different rental options and volume discounts from the perspective of a shipping company.

\section{Problem analysis}

Before presenting a mixed integer program for the VRPPCdR, we specify the problem in detail and introduce notations in the following.

\subsection{Problem specification and notation}

The VRPPCdR considers four different transportation options for the delivery of products from the shipper's depot to customer locations. Basic planning tasks include, first, the selection of the transportation option for each customer to be served; second, the clustering of a subset of the set of customers to one available (own or rented) vehicle or to one available common 
carrier; and third, the routing of private and rented vehicles as necessary. The objective is to minimize total delivery (transportation) costs. Therefore, $C$ represents the set of all vertices $h, i, j=0, \ldots, n$ (representing customers and the depot, which is indexed by 0 ) and $C^{n}$, which represents the set of $n$ customers where $n=\left|C^{n}\right|$. The distance between two vertices is defined by $d_{i j}$ and the demand quantity of customer $i$ by $q_{i}$.

The first transportation option is self-fulfillment by the heterogeneous private vehicle fleet of the shipping company. This set of $m$ vehicles is denoted by $V(m=|V|)$. The heterogeneous, limited capacity for each vehicle $k$ is defined by $c a p_{k}$ and the variable cost rate per distance unit is defined by $c_{k}$. The route of each of these private vehicles must start and end at the shipper's depot. Although each of the own vehicles is associated with fixed costs $c_{k}^{F i x}$, we assume that these costs are not relevant in the short-term planning process but only when investigating different fleet sizes. This assumption is in line with Krajewska and Kopfer (2009) but opposes other approaches (e.g., Chu, 2005 or Bolduc et al., 2007). Nevertheless, to compare, it would be easy to incorporate fixed costs as an additive term in the objective function.

To increase the transportation flexibility of the shipping company to fulfill FTL deliveries, we consider two options for exclusive vehicle rental. The subcontractor providing the first rental option charges a fee based on the distance of the route covered by one of his vehicles $k$. Therefore, a cost rate $c_{k}^{D i s t}$ per distance unit for each of the $m^{\prime}$ vehicles of set $R^{\prime}\left(m^{\prime}=\left|R^{\prime}\right|\right)$ is given. The fixed vehicle costs of this subcontractor are partially settled by the cost rate $c_{k}^{\text {Dist }}$. Therefore, $c_{k}^{\text {Dist }}>c_{k}$ strictly holds. To assert a minimum contribution to fixed costs, a minimum distance $d_{k}^{\text {Min }}$ per rentable vehicle $k \in R^{\prime}$ is defined by the subcontractor. Fees for the second rental option are accounted for on a daily basis. The subcontractor, who is paid on this daily basis, offers $m^{\prime \prime}$ vehicles from set $R^{\prime \prime}\left(m^{\prime \prime}=\left|R^{\prime \prime}\right|\right)$. These vehicles are charged for at cost rate $c_{k}^{\text {Day }}$ if vehicle $k$ is utilized in the resulting delivery plan. Here, the maximum utilization of vehicle $k$ is limited by maximum distance $d_{k}^{\text {Max }}$ per vehicle $k \in R^{\prime \prime}$. A maximum travel time could also be specified and converted to a maximum distance using standard travel speeds. All rentable vehicles also have a limited capacity $\mathrm{cap}_{k}$ and must start and end their routes at the shipper's depot. Because we assume that the company offering rental options is closely located to the shipper's depot, we neglect distances and travel times between the shipper's depot and these companies.

For all vehicles $k=1, \ldots, m, \ldots, m+m^{\prime}, \ldots, m+m^{\prime}+m^{\prime \prime}$ from the sets $V, R^{\prime}$, and $R^{\prime \prime}$, a subset of customers must be assigned (clustering), and a travel route must be determined (routing). As a result, those routes define the transportation costs either by the total traveled distance of the route $\left(k \in V \cup R^{\prime}\right)$ or by whether vehicle $k$ is utilized at all $\left(k \in R^{\prime \prime}\right)$.

The fourth transportation option is offered by a set $E$ of $e=|E|$ common LTL carriers (logistic service providers or freight forwarding companies). It is assumed that these carriers do not have any capacity limits but accept every subcontracted volume. Each of these carriers $l$ defines individual parameters that are used to calculate the transportation costs for this type of subcontracting. In most publications, the cost for delivering a product to the customer only depends on the customer's locations and is represented by a linear function of the distance between the customer and depot (e.g., Chu, 2005 or Bolduc et al., 2007). In contrast, Vahrenkamp (2011) states that a reasonable cost function must increase in both the quantity of delivered units and the distance from depot to customer location (as is common in public postal services). Krajewska and Kopfer (2009) also use this approach for the ITPP, defining a freight function by the weight of the cargo $q_{i j}$, the distance $d_{i j}$ between two vertices $i$ and $j$, a constant freight rate $c f r$, and the parameter $\lambda \in(0,1)$ :

$$
\operatorname{fr}\left(d_{i j}, q_{i j}\right)=c f r \cdot\left(d_{i j} \cdot\left(q_{i j}\right)^{\lambda}\right)^{1-\lambda}
$$

Transferring this freight function (1) to the VRPPCdR to determine the costs for serving customer $i \in C^{n}$ with one of the heterogeneous external carriers $l \in E$, the following (non-linear) cost function is used:

$$
c f_{i l}=\operatorname{tr}_{l} \cdot\left(d_{0 i} \cdot\left(q_{i}\right)^{\lambda_{l}}\right)^{1-\lambda_{l}}=t r_{l} \cdot \omega_{i l}
$$

In cost function (2), $t r_{l}$ represents a constant tariff rate, and parameter $\lambda_{l} \in(0,1)$ defines the slope of the function $\left(\omega_{i l}\right.$ is introduced to increase readability in the following): For $\lambda_{l}=1$, the cost function is a horizontal line at the value of $t_{l}$. For $\lambda_{l}=0$, the cost function is a linear function with slope $t r_{l}$ and is independent of quantity $q_{i}$. Therefore, the usually used cost function (e.g., by Chu, 2005 or Bolduc et al., 2008) is a special case of (2) in which $\lambda=0$. The actual parameter values of cost function (2) and also of the volume discounts described in the following strongly depend on the goals (e.g., to acquire new customers) and the cost estimation of the common carrier (for the estimation of distribution costs see e.g., Turkensteen and Klose, 2012 or Sun et al., 2015).

In addition to the extension of the VRPPC by rental options and cost function (2), we further consider all-unit volume discounts offered by LTL carriers. Here, we assume that the tariff rate $t_{l}$ itself is a function depending on the total delivery quantity outsourced to one of the LTL carriers $l \in E$. Therefore, the volume discount $\delta_{l}$ granted by carrier $l$ is represented by a monotonically increasing function of the total quantity outsourced to carrier $l$ (defined by the set $O_{l}$ of customers assigned to carrier $l$ ). Instead of this function, also a piecewise linear function representing incremental freight discounts could be easily incorporated (like in Sheen and Tsao, 2007; Tsao and Lu, 2012, or Stenger et al., 2013a). In this paper, the discount is defined by a discount factor $d f_{l}$.

$$
\delta_{l}=d f_{l} \cdot \sum_{i \in O_{l}} q_{i}
$$


To prevent volume discounts increasing to a level above the undiscounted tariff rate $t_{l}$, a minimal transportation rate $t r_{l}^{M i n}$ for every carrier $l$ is used. Özkaya et al. (2010) discuss a similar approach to determine prices of LTL shipments (see also Stenger et al., 2013a). The following cost function integrates all of the previous considerations:

$$
\left.c f_{i l}=\max \left\{\operatorname{tr}_{l}-d f_{l} \cdot q_{i} \sum_{i \in O_{l}}\right), \operatorname{tr}_{l}^{\text {Min }}\right\} \cdot\left(d_{0 i} \cdot\left(q_{i}\right)^{\lambda_{l}}\right)^{1-\lambda_{l}}=\max \left\{\operatorname{tr}_{l}-\delta_{l}, \operatorname{tr}_{l}^{\text {Min }}\right\} \cdot \omega_{i l}
$$

Unfortunately, the necessary maximum function (to guarantee a minimum transportation rate for the common carrier) leads to a non-linear cost function. This function will be linearized in the following to be able to provide a reasonably solvable mathematical formulation (the non-linearity of $\omega_{i l}$ is not important in this context, because as a coefficient, it is applied independently of the selection and clustering decisions).

\subsection{Mixed integer program}

The objective function of the VRPPCdR consists of four parts, one for each of the transportation options. This approach has been chosen because of the multiple carriers with individual cost parameters and rental options. Thus, we do not expand the formulation for the heterogeneous VRP (HVRP) as proposed by Bolduc et al. (2008), but follow the modeling approach presented in Krajewska and Kopfer (2009).

Within the mathematical formulation, the following decision variables are used:

$$
\begin{aligned}
& X_{i j k}= \begin{cases}1 & \text { if vehicle } k \in V \cup R^{\prime} \cup R^{\prime \prime} \text { visits vertex } j \text { immediately after vertex } i \\
0 & \text { otherwise }\end{cases} \\
& X_{k}= \begin{cases}1 & \text { if vehicle } k \in R^{\prime \prime} \text { is utilized(leaves the depot) } \\
0 & \text { otherwise }\end{cases} \\
& Y_{i k}= \begin{cases}1 & \text { if vehicle } k \in V \cup R^{\prime} \cup R^{\prime \prime} \text { visits vertex } i \\
0 & \text { otherwise }\end{cases} \\
& Z_{i l}= \begin{cases}1 & \text { if customer } i \text { is assigned to carrier } l \in E \\
0 & \text { otherwise }\end{cases}
\end{aligned}
$$

Based on these variables and the redefinition of (3) to $\delta_{l}=d f_{l} \sum_{j \in C^{n}} Z_{j l} \cdot q_{j}$, the objective function can be defined as follows:

$$
\begin{aligned}
& \min \sum_{k \in V} c_{k} \sum_{i \in C} \sum_{j \in C, i \neq j} X_{i j k} \cdot d_{i j}+\sum_{k \in R^{\prime}} c_{k}^{D i s t} \sum_{i \in C} \sum_{j \in C, i \neq j} X_{i j k} \cdot d_{i j}+\sum_{k \in R^{\prime \prime}} X_{k} \cdot c_{k}^{D a y} \\
& +\sum_{l \in E} \sum_{i \in C^{n}} Z_{i l} \cdot \max \left\{\operatorname{tr}_{l}-\left(d f_{l} \cdot \sum_{j \in C^{n}} Z_{j l} \cdot q_{j}\right), \operatorname{tr} r_{l}^{\text {Min }}\right\} \cdot\left(d_{0 i} \cdot\left(q_{i}\right)^{\lambda_{l}}\right)^{1-\lambda_{l}}
\end{aligned}
$$

Because the maximum operator of the cost function for the external carriers is not linear, this part of the objective function (5) is linearized to allow use of linear (standard) solution techniques to solve at least small VRPPCdR instances. This linearization (illustrated in detail in Appendix A) leads to the subsequent MIP for the VRPPCdR. The linear objective function is stated in formula (6), and the constraints are given in formulas (7)-(21). Formulas (22)-(25) define the domain of the decision variables.

$$
\begin{aligned}
& \left.\min \sum_{k \in V} c_{k} \sum_{i \in C} \sum_{j \in C, i \neq j} X_{i j k} \cdot d_{i j}+\sum_{k \in R^{\prime}} c_{k}^{D i s t} \sum_{i \in C} \sum_{j \in C, i \neq j} X_{i j k} \cdot d_{i j}+\sum_{k \in R^{\prime \prime}} X_{k} \cdot c_{k}^{D a y}+\sum_{l \in E} t r_{l} \sum_{i \in C^{n}} P_{i l} \cdot \omega_{i l}\right) \\
& \left.-\left(\sum_{l \in E} d f_{l} \sum_{i \in C^{n}} \sum_{j \in C^{n}} Q_{i j l} \cdot \omega_{i l} \cdot q_{j}\right)+\sum_{l \in E} \operatorname{tr} r_{l}^{M i n} \sum_{i \in C^{n}}\left(Z_{i l}-P_{i l}\right) \cdot \omega_{i l}\right)
\end{aligned}
$$

subject to

$$
\begin{aligned}
& \sum_{i \in C^{n}} Y_{i k} \cdot q_{i} \leqslant \operatorname{cap}_{k} \quad \forall k \in V \cup R^{\prime} \cup R^{\prime \prime} \\
& \left.\left.\sum_{k \in V \cup R^{\prime} \cup R^{\prime \prime}} Y_{i k}\right)+\sum_{l \in E} Z_{i l}\right)=1 \quad \forall i \in C^{n} \\
& \sum_{i \in C} X_{i j k}=Y_{j k} \quad \forall k \in V \cup R^{\prime} \cup R^{\prime \prime}, j \in C
\end{aligned}
$$




$$
\begin{aligned}
& \sum_{j \in C} X_{i j k}=Y_{i k} \quad \forall k \in V \cup R^{\prime} \cup R^{\prime \prime}, i \in C \\
& Y_{0 k} \leqslant 1 \quad \forall k \in V \cup R^{\prime} \cup R^{\prime \prime} \\
& \sum_{j \in C^{n}} X_{0 j k}=X_{k} \quad \forall k \in R^{\prime \prime} \\
& \sum_{i \in C} \sum_{j \in C, i \neq j} d_{i j} \cdot X_{i j k} \leqslant d_{k}^{M a x} \quad \forall k \in R^{\prime \prime} \\
& \sum_{i \in C} \sum_{j \in C, i \neq j} d_{i j} \cdot X_{i j k} \geqslant d_{k}^{M i n} \cdot Y_{0 k} \quad \forall k \in R^{\prime} \\
& \left.\left.\quad \sum_{i} \leqslant \quad Y_{i k} \cdot \operatorname{cap}_{k}\right)+\sum_{l \in V \cup R^{\prime} \cup R^{\prime \prime}} Z_{i l} \cdot q_{i}\right) \quad \forall i \in C^{n} \\
& \left.U_{j} \geqslant U_{i}+q_{i}+\quad \sum_{k \in V \cup R^{\prime} \cup R^{\prime \prime}} X_{i j k} \cdot c a p_{k}\right)- \\
& \left.\left.U_{i} \geqslant q_{i} \quad \forall i \in C^{n} \quad \sum_{k \in V \cup R^{\prime} \cup R^{\prime \prime}} Y_{i k} \cdot \operatorname{cap}_{k}\right)-\quad Z_{l \in E} Z_{i l} \cdot q_{i}\right) \quad \forall i, j \in C^{n} \text { with } i \neq j \\
& Z_{i l}-P_{i l} \geqslant 0 \quad \forall i \in C^{n}, l \in E \\
& Z_{i l}+Z_{j l}+P_{i l}-3 Q_{i j l} \geqslant 0 \quad \forall i, j \in C^{n}, l \in E \\
& \left(\operatorname{tr}_{l} \cdot Z_{i l}-\delta_{l}\right)-\operatorname{tr}_{l}^{M i n} \leqslant P_{i l} \cdot M \quad \forall i \in C^{n}, l \in E \\
& \left(\operatorname{tr}_{l}-\delta_{l}\right)-\operatorname{tr}_{l}^{M i n} \geqslant\left(P_{i l}-1\right) \cdot M \quad \forall i \in C^{n}, l \in E \\
& X_{i j k}, Y_{i k} \in\{0,1\} \quad \forall k \in V \cup R^{\prime} \cup R^{\prime \prime}, i, j \in C \\
& X_{k} \in\{0,1\} \quad \forall k \in R^{\prime \prime} \\
& P_{i l}, Q_{i j l} \in\{0,1\} \quad \forall i \in C^{n}, l \in E \\
& U_{i} \in \mathbb{Z}_{2} \geqslant 0 \quad \forall i \in C^{n}
\end{aligned}
$$

The capacity constraints (7) are defined for all own and rented vehicles. Constraint set (8) represents the single-delivery constraint. Constraint sets (9) and (10) ensure that the same vehicle enters and leaves a customer exactly once, and constraint set (11) ensures that all routed vehicles start from the depot. Constraint set (12) establishes the relationship between the "renting variable" $X_{k}$ and the "routing variable" $X_{i j k}$. The maximal route length for vehicles rented on a daily basis is obeyed by (13), whereas (14) ensures the minimal distance of routes performed by vehicles charged per distance unit. The next constraint sets (15)-(17) are sub-tour elimination constraints. Those are Miller-Tucker-Zemlin (MTZ) constraints (Miller et al., 1960) and are modified from the $\mathrm{HVRP}_{3}$ formulation presented in Yaman (2006). Note, that we also tested formulations using the sub-tour elimination constraints as first proposed in Dantzig et al. (1954), but we found that for the VRPPCdR, the MTZ constraints provide a higher solution quality in shorter computation times. Constraint sets (18)-(21) are used for the linearization of volume discounts. Constraint set (18) allows $P_{i l}$ only being equal to 1 if $Z_{i l}=1$, but permits $P_{i l}=0$. The constraint set (19) works similarly for $Q_{i j l}$ : All three variables $Z_{i l}, Z_{j l}$, and $B_{l}$ must be equal to 1 to permit $Q_{i j l}=1$. Due to the minimization objective, the constraints defined by (20) and (21) prohibit $P_{i l}=1$ if $t r_{l}-\delta_{l}<t r_{l}^{M i n}$, and vice versa. Here, the character $M$ represents a sufficiently large number. For the formulation at hand, $M$ can be approximated by $M \geqslant \max \left(\operatorname{tr}_{l}\right)$.

\section{Solution method}

Because the VRPPCdR generalizes the NP-hard VRP and VRPPC (see Lenstra and Rinnooy Kan, 1981; Stenger et al., 2013b), it is also NP-hard. Thus, we develop three heuristic solution methods able to solve virtually any size of problem instances. Concerning the problem at hand, we especially focus on the development of very efficient heuristics, i.e., heuristics that are very fast in terms of computation time and achieve a sufficient good solution quality.

Starting with an initial solution calculated by a problem-specific construction heuristic, all three proposed heuristics are based on the principles of Variable Neighborhood Search (VNS; first described in Mladenović and Hansen, 1997). VNS is applied because several papers show the suitability of VNS to solve VRPs and closely related problems (e.g., Hertz and Mittaz, 2001; Bräysy, 2003; Polacek et al., 2004; Kytöjoki et al., 2007; Stenger et al., 2013b). Generally, VNS is a metaheuristic embedding a local search mechanism within a framework that uses multiple, varying neighborhood structures to exploit local optima and to escape from them (Hansen et al., 2010). These neighborhood structures are also used to define a solution perturbation mechanism ("shaking").

In addition to this well-known features of VNS, a completely new enhancing mechanism for VNS-based heuristics is introduced: "explicit shaking". This enhancement is meant to explicitly interrupt the search procedure in already exhaustively searched areas of the solution space and thus, to direct the search process to other areas. The actual implementation of this explicit shaking mechanism is of course problem specific but the mechanism itself could be transferred to any VNS-based 
solution method. Furthermore, we introduce a distance proportionate vehicle selection mechanism to increase the likelihood of the local search for improvements.

All components of the proposed solution methods for the VRPPCdR, the problem-specific construction heuristic, the (new) VNS variants, the neighborhood structures and local search procedures, and the new explicit shaking mechanism are described in detail in the following sections.

\subsection{Initial construction heuristic}

The proposed construction heuristic for the initialization is a problem specific adaption of the procedure described in Bolduc et al. (2007). It integrates the two rental options and is able to handle multiple external carriers with individual parameters. Furthermore, we have modified the used savings heuristic (Clarke and Wright, 1964) to the specific requirements defined by the two rental options. Additionally, the route construction procedure is terminated before the load of a vehicle $k$ reaches $100 \%$ of its capacity $\left(c a p_{k}\right)$. The reason for this is that the succeeding solution improvement heuristic performs better if there is a certain amount of remaining capacity in each vehicle (also stated by Kytöjoki et al., 2007). This initial vehicle utilization is controlled by the parameter $\sigma \in \mathbb{R}(0<\sigma \leqslant 1) . \sigma$ can be considered the percentage initial maximum utilization of every vehicle of the private fleet. The pseudocode Listing 1 illustrates the main course of action of the construction heuristic.

The heuristic starts with the calculation of the total customer demand (Listing 1-1) and the total capacity provided by the vehicles of the private fleet and all rentable vehicles (Listing 1-2; adjusted by $\sigma$ ). Here, we use the total capacity of the private fleet and all rentable vehicles because we follow the assumption that (full) truckloads lead to lower costs than LTL subcontracting for individual shipping.

Then, the set of customers $C^{n}$ is sorted in ascending order by their mean external delivery costs (Listing 1-3). Because not only one external carrier is available but multiple ones, mean external delivery costs $c_{i}^{\text {Mean }}$ are calculated for each customer $i$ (naturally, volume discounts are not considered here):

$$
c_{i}^{\text {Mean }}=\frac{1}{e} \cdot \sum_{l \in E} c f_{i l}
$$

Based on this ordering, a separation index $h$ is calculated (Listing 1-4; see Bolduc et al., 2007) and $C^{n}$ is divided into the sets $C^{V, R}$ and $C^{E}$ (Listing 1-5) such that $C^{E}$ contains the customers with the lowest mean external delivery costs and $C^{V, R}$ contains at most as many customers as can be served with respect to the adjusted capacity of the available vehicles in $V, R^{\prime}$, and $R^{\prime \prime}$.

The following modified sequential savings heuristic (Listing 1-6) constructs route by route as the original savings heuristic (Clarke and Wright, 1964) but only as many routes as vehicles (including both the private fleet and the rentable vehicles) are available. The heuristic starts with the largest unused (i.e., no route is assigned) vehicle $k$ and constructs feasible routes with respect to the adjusted capacity $\left(c a p_{k} \cdot \sigma\right)$ and, where necessary, with respect to minimum distance $d_{k}^{\text {Min }}$ (if $k \in R^{\prime}$ ) or to maximum distance $d_{k}^{\text {Max }}$ (if $k \in R^{\prime \prime}$ ). Once all routes are constructed, the feasible route with the largest assigned demand quantity is assigned to $k$, all served customers are removed from $C^{V, R}$, and $k$ is marked as in use. This procedure is repeated until either all customers are assigned to routes or no vehicle from $V, R^{\prime}$, or $R^{\prime \prime}$ is unused. Potentially unassigned customers are added to $C^{E}$ (Listing 1-7).

Having all routes constructed and assigned, these routes are optimized (Listing 1-8) by Helsgaun's LKH-2 implementation (see Helsgaun, 2000; Helsgaun, 2009; available at http://www.akira.ruc.dk/ keld/research/LKH/) of the Lin-Kernighan algo-

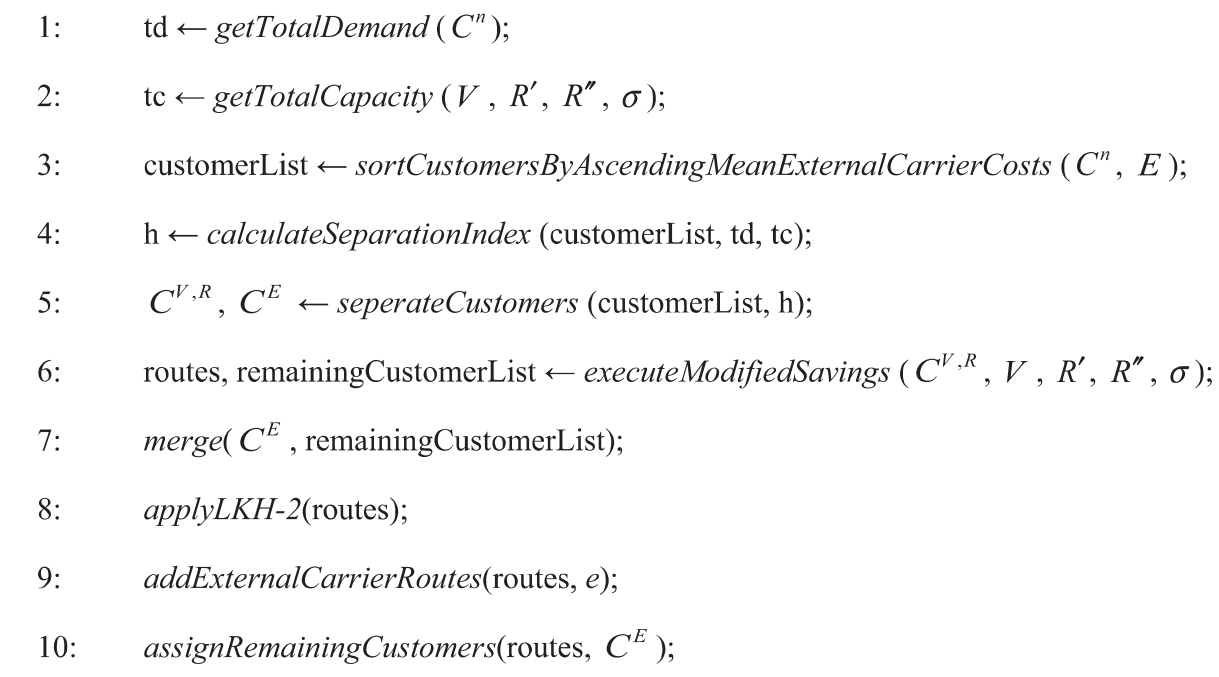

Listing 1. Main course of action of the construction heuristic. 


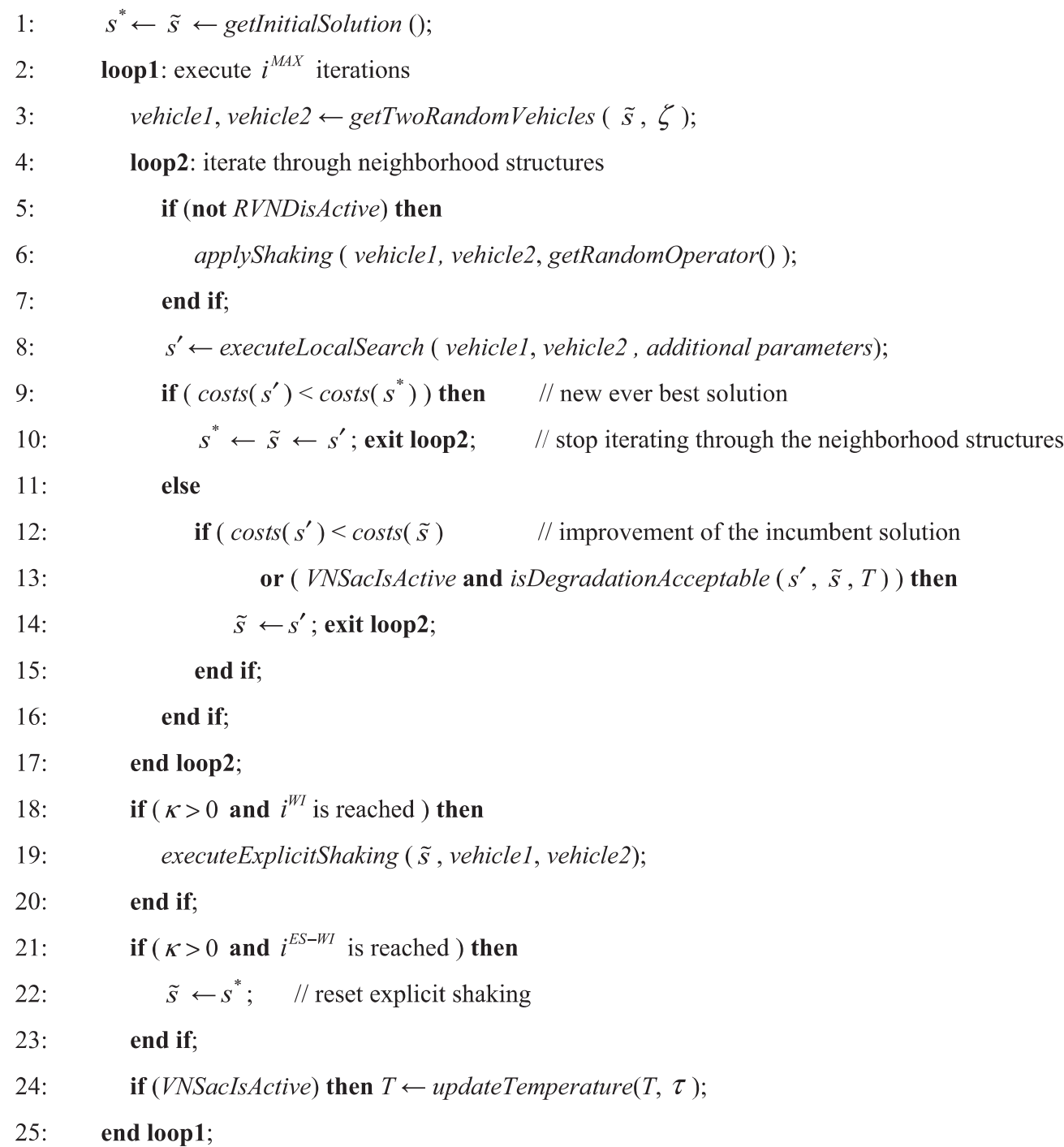

Listing 2. Main course of action of the VNS-variants.

rithm (Lin and Kernighan, 1973). This heuristic is used because it is one of the most powerful heuristics to solve Traveling Salesman Problems (Johnson and McGeoch, 2007; Karapetyan and Gutin, 2011) and, thus, for intra-route optimization.

To model subcontracting with external carriers, we use the concept of "virtual vehicles" (as, e.g., proposed by Bolduc et al., 2007; Stenger et al., 2013b), and $e$ virtual routes are added to the list of "real" routes (Listing 1-9). This concept of virtual vehicles is used by all proposed solution methods. Finally, all customers from $C^{E}$ are assigned to the external carrier with the lowest cost (calculated by cost function (2)) for serving this customer (Listing 1-10). Because all constraints are considered throughout the construction of the solution, the initial solution is always feasible. This feasibility of a solution is also ensured by all subsequently described solution transformations, and only feasible solutions are considered by the solution methods.

\subsection{Main course of action of the VNS-variants}

To solve the VRPPCdR, we propose a basic VNS variant as described in Hansen et al. (2010), a randomized Variable Neighborhood Descent (RVND) variant, and a VNS variant (named VNSac) that uses an acceptance criterion such as the Simulated Annealing (SA) metaheuristic. The RVND variant is inspired by the RandVND metaheuristic proposed in Gahm et al. (2014) and extends the basic Variable Neighborhood Descent (VND; Hansen et al., 2010) method by a random component. For all variants proposed here, we randomly select two vehicles (routes) before starting the neighborhood search. Therefore, we do not investigate all two-route combinations as was done by Kytöjoki et al. (2007) but only two randomly selected ones. Due to the random selection procedure, the RVND-variant is no longer deterministic but stochastic. The VNSac variant is developed because Hemmelmayr et al. (2009) reported a superior behavior of their SA-based VNS approach compared with their Skewed VNS and their VNS approach incorporating an acceptance criterion based on the Threshold Accepting concept. The main course of action for all three variants is described by the pseudocode in Listing 2. 
The course starts with the initialization of the best known solution $s^{*}$ and the incumbent solution $\tilde{s}$ by the construction heuristic. After this initialization, the main control loop is traversed for a maximum number of iterations $i^{\text {MAX }}$ (Listing 2-2). The maximum number of iterations can be used by the decision maker to balance the trade-off between solution quality and computation time.

Regardless of the specified variant, each iteration starts with the random selection of two different vehicles/routes (Listing 2-3). Here, to increase the likelihood for improving moves within the subsequent local search, a new distance proportionate vehicle selection mechanism is introduced (see Section 4.4 for details).

The inner control loop (Listing 2-4) iterates through the defined neighborhood structures $n s_{1}$ to $n s_{5}$ as described in detail in Section 4.3 (see Table 1). Then, for the two variants VNS and VNSac, a shaking step is performed by a randomly selected operator (from the currently active neighborhood structure) and the two randomly selected vehicles (Listing 2-6). Because the RVND variant bases on VND, no shaking step is executed (see Hansen et al., 2010).

The current ("shaked") solution is then improved by a specified local search procedure and returns the new solution $s^{\prime}$ (Listing 2-8; the local search procedure is described in the following section).

When the costs of solution $s^{\prime}$ (calculated by cost function (6)) are less than that of the best known solution $s^{*}$, solution $s^{\prime}$ becomes $s^{*}$ and also the new incumbent solution $\tilde{s}$ and iterating through the neighborhood structures is terminated (Listing $2-9 . .10$ ). If only the costs of incumbent solution $\tilde{s}$ are improved by $s^{\prime}$ (Listing 2-12) or the VNSac variant is active and the cost degradation is acceptable (Listing 2-13), then the new solution $s^{\prime}$ becomes $\tilde{s}$ and iterating through the neighborhood structures is also terminated (Listing 2-14). The acceptance probability of non-improving solutions is defined by $\exp \left(-\left(\operatorname{costs}\left(s^{\prime \prime}\right)-\operatorname{costs}(\tilde{s})\right) / \mathrm{T}\right)$, where T represents the (current) temperature. In any other case, iterating through the neighborhood structure is continued.

If the explicit shaking mechanism is activated at all $(\kappa>0)$ and a specified number of iterations without improvement $i^{W I}$ is reached, an "explicit shaking" mechanism as described in Section 4.5 is executed (Listing 2-18. . .20). To avoid the exploration of unpromising parts of the solution space when explicit shaking is activated, a mechanism is available to reset the incumbent solution $\tilde{s}$ to the best known solution $s^{*}$ if no improvement of $s^{*}$ can be achieved within $i^{E S-W I}$ iterations (Listing $2-21 . .23)$.

The last step within loop1 is the update of the temperature for the VNSac variant (Listing 2-24). To update the temperature, we use a linear cooling scheme based on cooling factor $\tau: T_{t+1}=T_{t} \cdot \tau$. Different cooling schemes (exponential cooling and step functions) have also been considered, but linear cooling with $\tau=0.3$ provided the best results.

\subsection{Neighborhood structures and local search procedure}

The most important aspects when applying VNS to a specific problem are the definition and number of appropriate neighborhood structures (Listing 2 loop2), the order in which these neighborhoods are investigated, the strategy that is used for changing neighborhoods, and the local search procedure (Hansen and Mladenović, 2001).

Neighborhood structures and also the local search procedures are based on transformation rules, which define the transformation of a solution to obtain another solution. For the VRPPCdR, we use increasingly large neighborhood structures with changing local search mechanisms based on $\lambda$-interchanges (Osman, 1993). Each neighborhood structure is defined by a set of operators that define the $\lambda$-interchanges and the extraction/insertion (e/i) mechanism to use. According to Osman (1993), shifting or transferring one customer from one route to another is defined by the $(1,0)$ and $(0,1)$ operator, respectively. Specifically, the operator $(1,0)$ means that a single customer is extracted from one route and inserted into another route. Shifting two customers is defined by $(2,0)$ and $(0,2)$, shifting three by $(3,0)$ and $(0,3)$, and so on. Interchanging or swapping customers between two routes is defined by operators such as $(1,1)$ and $(2,2)$. In contrast to Stenger et al. (2013b), we only consider two routes for inter-route optimization because this approach is also used by the very performant VNS metaheuristic presented in Kytöjoki et al. (2007). To improve the efficiency of the inter-route optimization, we introduce a new mechanism to randomly select these two routes. This mechanism is described in Section 4.4.

To extract and insert customers from and into a route, we use two local-search mechanisms: "sequence-e/i" and "single$\mathrm{e} / \mathrm{i}$ ". To transfer promising sequences between two routes, the sequence e/i-mechanism extracts customers in sequence and inserts this sequence into the other route, preserving the sequence. In contrast, the single e/i-mechanism extracts randomly chosen customers and inserts them individually. With this mechanism, the sequence of both routes is broken up. To insert either the complete sequence of customers or all customers individually, the position with the lowest cost is calculated for insertion. Of course, if customers are going to be transferred to virtual routes, no insertion position is calculated but only the costs. Due to the heterogeneous vehicle fleet considered by the VRPPCdR, it is not possible to use the very fast cost estimation procedure as described in Bolduc et al. (2008). After applying one of the operators, the routes are tested for cost improvements and for feasibility and, if they are feasible, an intra-route optimization step is performed by 2-opt exchanges (Lin, 1965). We use 2-opt exchanges because tests have shown that more-sophisticated methods for intra-route optimization such as the outstanding LKH-2 heuristic have only marginal positive effects on the solution quality but significantly increase computation time. 
Table 1

Neighborhood structures.

\begin{tabular}{lll}
\hline Neighborhood structure & e/i-mechanism & $\lambda$-operator sets \\
\hline$n s_{1}$ & Single-insertion & $\{(1,0),(0,1),(1,1)\}$ \\
$n s_{2}$ & Sequence-insertion & $\{(2,0),(0,2),(2,1),(1,2),(2,2),(3,0),(0,3),(3,1),(1,3),(3,2),(2,3),(3,3)\}$ \\
$n s_{3}$ & Single-insertion & \\
$n s_{4}$ & Sequence-insertion & $\{(4,0),(0,4),(4,1),(1,4),(4,2),(2,4),(4,3),(3,4),(4,4),(5,0),(0,5),(5,1)$, \\
$n s_{5}$ & Single-insertion & $(1,5),(5,2),(2,5),(5,3),(3,5),(5,4),(4,5),(5,5)\}$ \\
\hline
\end{tabular}

Based on preliminary tests, we also identified the sequence of five neighborhood structures $n s_{1}$ to $n s_{5}$ listed in Table 1 as most appropriate concerning solution quality and computation time.

Input data and parameters for the local search procedure applied to each neighborhood structure are two routes, one operator, the e/i-mechanism and the improvement strategy. The improvement strategy parameter $\psi$ defines the manner in which solutions will be selected to become the incumbent solution. The strategies "best improvement/steepest decent (BI)" and "first improvement/first descent (FI)" are used (for details see, e.g., Hansen et al., 2010). The BI-strategy evaluates all possible solutions defined by one neighborhood structure and returns the best of these solutions. In contrast, using the FI-strategy, the evaluation of a neighborhood structure stops as soon as some improvement is detected. Obviously, this parameter influences the number of neighboring solutions to be explored and thus, has a strong influence on the computation time. This influence on the computation time and on the solution quality will be analyzed in detail within the computational analysis in Section 5.

\subsection{Vehicle selection mechanism}

Two mechanisms are used to select the two vehicles for inter-route optimization (local search). The binary parameter $\zeta$ controls their application. The first mechanism $(\zeta=0)$ randomly selects two vehicles from all available real and virtual vehicles. The second mechanism $(\zeta=1)$ also selects vehicles randomly, however, if the first selected vehicle $v 1$ is a real vehicle (in one of the sets $V, R^{\prime}$, or $R^{\prime \prime}$ ), then we apply a distance proportionate mechanism for the selection of the second vehicle $v 2$. If the first vehicle represents a common carrier, the second one is randomly selected from all other real or virtual available vehicles. In this case, all vehicles have a uniform probability to be selected for first or second vehicle. This procedure is inspired from fitness proportional selection often used in Genetic Algorithms (see e.g., Grefenstette, 2000).

Basic idea of the distance proportionate selection mechanism is to guide the selection of vehicles for inter-route optimization in a way that increases the probability of finding an improvement. Vehicle routes in close proximity to the first selected vehicle (route) have a greater probability for a successful inter-route improvement move than routes that are far away. To determine the proximity of two routes, the Euclidean distance of their center points $c p_{v 1}$ and $c p_{v 2}$ is used. The center point $c p_{v}$ of a route (vehicle) is calculated by the centroid of the axis-aligned minimum bounding rectangle (AAMBR) of all customers on the route (Fig. 1 illustrates the calculation of Cartesian coordinates of the routes' center points). Of course, there are more (sophisticated) ways to calculate a center of a route (like the geometric median or solving a p-median problem). However, we decided for the AAMBR due to its low computational effort. For all common carriers, we use the mean distance of the real vehicles.

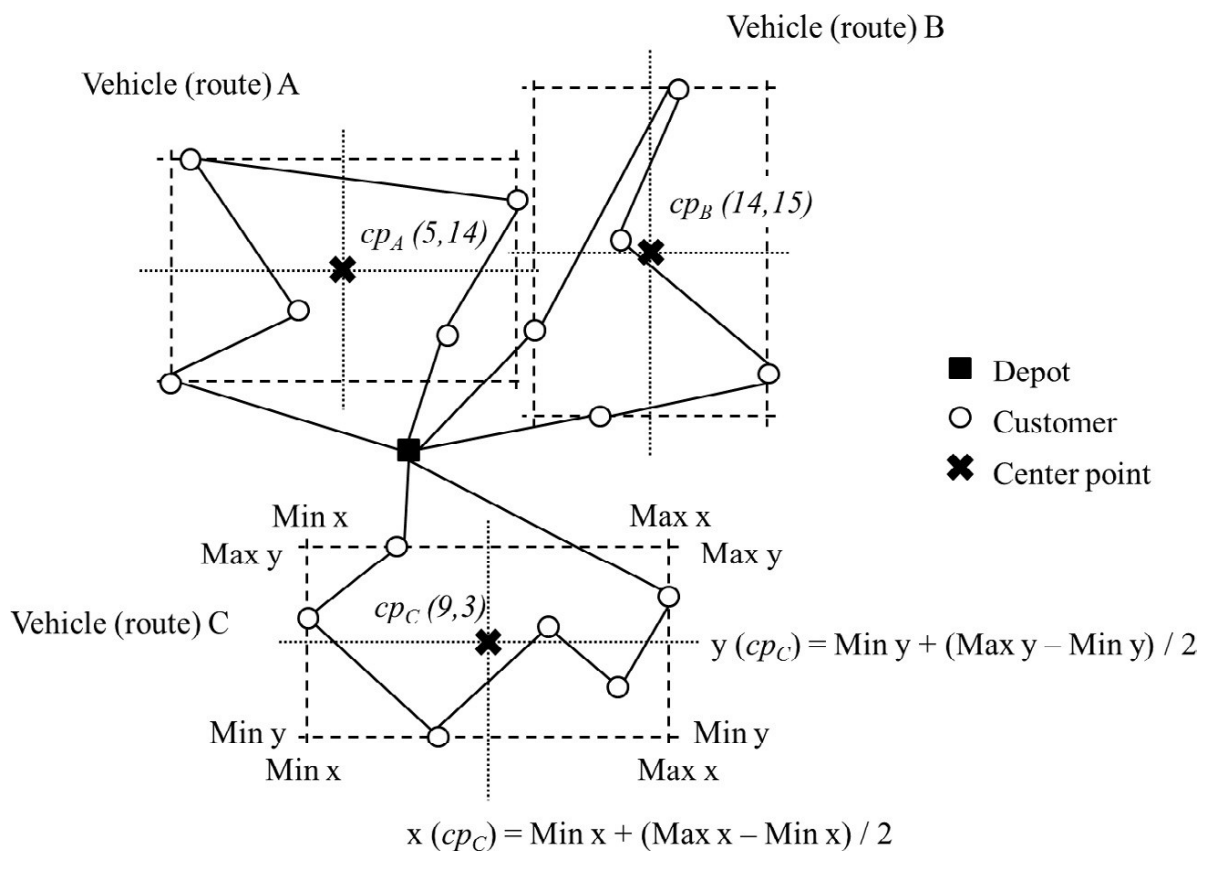

Fig. 1. Route center calculation with AAMBR. 
The intended effect can be best illustrated considering the vehicle routes in Fig. 1 . Assuming that vehicle $A$ with $c p_{A}(5,14)$ is selected as first vehicle, the probability that vehicle B with $c p_{B}(14,15)$ and dist $t_{A, B}=9.06$ is chosen as second vehicle should be higher than that of vehicle $C$ with $c p_{C}(9,3)$ and dist $t_{A, C}=11.7$. Therefore, the selection is based on a reciprocal distance proportionate mechanism. The effect of the distance based vehicle selection is analyzed in Section 5.2.

\subsection{Explicit shaking mechanism}

To support the exploration of the solution space and to avoid cycling in local optima, we use two additional mechanisms for solution perturbation. Because both mechanisms have a high intended perturbation effect, these rules are not used to define neighborhood structures but rather to define "explicit" shaking mechanisms (Listing 2-18 . .20).

The first of these very specific transformation rule is called "route swapping" (RS) because it swaps complete routes between two vehicles (similar to Krajewska and Kopfer, 2009). In contrast to Krajewska and Kopfer (2009), we swap routes not only between own and rented vehicles but between all (real and virtual) vehicles. The second transformation rule "route dividing" (RD) has also a very large perturbation effect and is only used if route swapping is not possible due to capacity or distance constraints (see (7), (13), and (14)). The RD-rule takes a real vehicle's route, extracts all customers, and inserts each of these customers to the external carrier with the lowest costs for serving this customer. After applying RD, one vehicle has an empty route and thus great potential for shifting customers to this route.

In principle, these explicit shaking mechanisms are applied whenever it is not possible to improve the best known solution $s^{*}$ for a certain number of iterations $i^{W I}$ (see Listing 2-20..22). To avoid a too exhaustive ineffective exploration of unpromising regions of the solution space after an explicit shaking step, we stop searching after a predefined maximum number of iterations $\left(i^{E S-W I}\right)$ without improvement of $s^{*}$, reset to the best known solution so far $\left(s^{*}\right)$, and perform another explicit shaking step (see Listing 2-23...25). The usage of the explicit shaking mechanisms is controlled by the parameter $\kappa \in \mathbb{R}$ (with $0 \leqslant \kappa<1$ ), whereby $\kappa=0$ has an additional meaning and avoids any explicit shaking. The additional parameter $\theta$ specifies whether the RD-transformation rule is used as an explicit shaking mechanism at all.

In the next section, the explicit shaking mechanisms RS and RD and the specific parameter settings of $\kappa$ and $\theta$ are analyzed with respect to their influence on the performance of the three VNS-variants. Furthermore, this analysis covers the influence of the improvement strategy parameter $\psi$ and the vehicle selection mechanism controlled by parameter $\zeta$.

\section{Computational analysis}

The computational analysis consists of two parts. In the first part, the performance of the proposed VNS-variants combined with different parameter settings is evaluated by the variants' solution quality and computation time. The second part assesses the economic benefits of considering multiple external carriers offering volume discounts and rental options. Furthermore, the particular circumstances of achieving these benefits are analyzed.

\subsection{Problem instances and basic parameter settings}

Before presenting the analysis, the used test instances and the basic parameter settings are described in this section. The first two sets of instances CHU and BOL contain the instances used in Chu (2005) and Bolduc et al. (2007). Unfortunately, we discovered some inconsistency using the objective values presented in these papers (we think caused by different rounding of the Euclidean distances); therefore, we calculated new objective values with the model described above and the commercial standard solver IBM CPLEX Optimizer 12.5 (the new reference values can be found in Appendix C, Table 14). To handle the rounding issue for these instances, we calculate the Euclidean distance $d_{i j} \in \mathbb{N}$ between two vertices $i$ and $j$ by their coordinates in advance by $d_{i j}=\left\lfloor\sqrt{\left(x_{i}-x_{j}\right)^{2}+\left(y_{i}-y_{j}\right)^{2}}\right\rfloor$.

The next four instance sets are based on the instances of Christofides and Eilon (1969) and Golden et al. (1998) and have been adapted by Bolduc et al. (2008) for the VRPPC. These four instance sets are named CE and G for instances with a homogeneous private vehicle fleet and $\mathrm{CE}-\mathrm{H}$ and $\mathrm{G}-\mathrm{H}$ for instances with a heterogeneous private vehicle fleet. The instances are available at http://www.mcbolduc.com/VRPPC/tests.htm. Reference values for instances in sets CE and G are taken from Stenger et al. (2013b); specifically, the objective values with their mean computation times as listed in "Table 4: Results on VRPPC Instances" in the paper of Stenger et al. (2013b) (but not the values in column "BKS" because computation times for these values are not given). For the instances in the sets $\mathrm{CE}-\mathrm{H}$ and $\mathrm{G}-\mathrm{H}$, we use the objective values with their mean computation times as listed in "Table 8: Heterogeneous instances" in the paper of Potvin and Naud (2011).

For the VRPPCdR, we define seven new instance sets based on the BOL-instance set. We combine each of the basic scenarios BOL-1 to BOL-5 (basic data can be found in Appendix C, Table 15) with a downsized private fleet (vehicle data are listed in Table 2) and seven "carrier and rental option"-scenarios (Table 5). The data of the private vehicles, the capacities and the cost factors, are the same as used in Bolduc et al. (2007). All used instances are available for download or can be requested by email. 
Table 2

Vehicle data of the private fleet $(k \in V)$.

\begin{tabular}{llll}
\hline Identifier & $c a p_{k}$ & $c_{k}$ & $c_{k}^{\text {Fix }}$ \\
\hline V1 & 40 & 1.5 & 60 \\
V2 & 75 & 1.5 & 120 \\
V3 & 110 & 1.5 & 150 \\
V4 & 100 & 1.5 & 140 \\
V5 & 4500 & 1.5 & 250 \\
V6 & 4000 & 1.5 & 200 \\
\hline
\end{tabular}

The parameters of rental options M1 to M3 (Table 3) and D1 and D2 (Table 4) are deduced from the original vehicles' parameters to obtain reasonable settings. Reasonable means that the cost factors for both rental options are specified according to the assumption that the cost rate per distance unit of the private fleet is always cheaper than the freight rate of any other delivery mode. In addition, this kind of parameter setting reflects the fact that shipping companies usually prefer the use of their own fleet before considering any type of subcontracting (not using the private vehicles is a kind of waste).

To get fitting parameter settings, the maximum capacity per rentable vehicle and the daily rate $c_{k}^{\text {Day }}$ are calculated based on the corresponding maximum values of all vehicles of the private fleet $R$ associated with the carrier and rental option scenario (the private vehicles data derive from Bolduc et al., 2007). Another factor that the cost factors $c_{k}^{\text {Dist }}$ are greater than are those of the private vehicles is that they contain proportional fixed costs. The $d_{k}^{\text {Min }}$ and $d_{k}^{\text {Max }}$ parameters are estimated based on the length of the optimal routes reported in Bolduc et al. (2007).

Table 3

Rental options paid on a mileage basis $\left(k \in R^{\prime}\right)$.

\begin{tabular}{llll}
\hline Identifier & $\operatorname{cap}_{k}$ & $c_{k}^{\text {Dist }}$ & $d_{k}^{\text {Min }}$ \\
\hline M1 & $\left\lfloor\max \left\{\operatorname{cap}_{k}: \forall k \in V\right\} \cdot 1.33\right\rfloor$ & 2.50 & 60 \\
M2 & $\max \left\{\operatorname{cap}_{k}: \forall k \in V\right\}$ & 2.25 & 60 \\
M3 & $\left\lfloor\max \left\{\operatorname{cap}_{k}: \forall k \in V\right\} \cdot 0.5\right\rfloor$ & 2.00 & 60 \\
\hline
\end{tabular}

Table 4

Rental options paid on a daily basis $\left(k \in R^{\prime \prime}\right)$.

\begin{tabular}{llll}
\hline Identifier & $\operatorname{cap}_{k}$ & $c_{k}^{\text {Day }}$ & $d_{k}^{\text {Max }}$ \\
\hline D1 & $\left\lfloor\max \left\{c a p_{k}: \forall k \in V\right\} \cdot 1.33\right\rfloor$ & $\left\lfloor\max \left\{c_{k}^{\text {Fix }}: \forall k \in V\right\} \cdot 2.66\right\rfloor$ & 120 \\
D2 & $\max \left\{\operatorname{cap}_{k}: \forall k \in V\right\}$ & $\max \left\{c_{k}^{\text {Fix }}: \forall k \in V\right\} \cdot 2$ & 120 \\
\hline
\end{tabular}

The seven carrier and rental option scenarios S1 to S7 contain different combinations of rental options and external carriers; however, S7 contains all possible options (Table 5).

Parameters of the four considered external carriers are shown in Table 6. In turn, these parameters are specified according to the assumption that subcontracting should generally be more expensive than self-fulfillment.

Table 5

Carrier and rental option scenarios.

\begin{tabular}{lll}
\hline Identifier & Rental options & Carriers \\
\hline S1 & & $\mathrm{C} 2, \mathrm{C} 3$ \\
S2 & $\mathrm{C} 1, \mathrm{C} 2, \mathrm{C} 3, \mathrm{C} 4$ \\
S3 & $\mathrm{M} 1, \mathrm{M} 3, \mathrm{D} 1, \mathrm{D} 2$ & $\mathrm{C} 1$ \\
S4 & $\mathrm{M} 2, \mathrm{M} 3, \mathrm{M} 3, \mathrm{D} 2$ & $\mathrm{C} 1$ \\
S5 & M2, D2 & $\mathrm{C} 2, \mathrm{C} 3, \mathrm{C} 4$ \\
S6 & M3, M3, D1, D2 & $\mathrm{C} 2, \mathrm{C} 3$ \\
S7 & M1, M2, M3, D1, D2 & $\mathrm{C} 1, \mathrm{C} 2, \mathrm{C} 3, \mathrm{C} 4$ \\
\hline
\end{tabular}

The first two external carriers C1 and C2 offer no volume discounts and have a constant tariff rate $\operatorname{tr}_{l}$. The tariff of C1 is independent of the delivered quantity, whereas $\mathrm{C} 2$ has a slope of $\lambda_{l}=0.4$. Carriers C 3 and C4 offer volume discounts and thus have a discount factor $d f_{l}$ and minimum tariff rate $\operatorname{tr}_{l}^{M i n}$ specified (the effect of the specified volume discount parameters for the carriers C3 and C4 is illustrated in Fig. 2 in Appendix B). 
Table 6

\begin{tabular}{lllll}
\multicolumn{1}{l}{ Carrier data $(l \in L)}$. \\
\hline Identifier & $t r_{l}$ & $\lambda_{l}$ & $d f_{l}$ & $t r_{l}^{\text {Min }}$ \\
\hline C1 & 4 & 0.0 & 0 & 0 \\
C2 & 7 & 0.4 & 0 & 0 \\
C3 & 7 & 0.2 & 0.02 & 3 \\
C4 & 5.5 & 0.2 & 0.015 & 4 \\
\hline
\end{tabular}

Altogether, these specifications lead to 35 problem instances (Table 7) grouped into five sets N1 to N5 (with respect to their basic data based on the BOL-instance set), each comprising the seven carrier and rental option scenarios S1 to S7 listed in Table 5.

Table 7

New problem instances for the VRPPCdR

\begin{tabular}{llll}
\hline Instance identifier & Private fleet & Carrier and rental option scenarios & Basic data (locations and order quantities) \\
\hline N1-01 . . N1-07 & V1 & S1-S7 & BOL-1 \\
N2-01 . . N2-07 & V2 & S1-S7 & BOL-2 \\
N3-01 . . N3-07 & V3, V4 & S1-S7 & BOL-3 \\
N4-01 . . N4-07 & V5 & S1-S7 & BOL-4 \\
N5-01 ... N5-07 & V5, V6 & S1-S7 & BOL-5 \\
\hline
\end{tabular}

Table 8

Parameters for the three VNS-variants (based on preliminary tests).

\begin{tabular}{|c|c|c|}
\hline Parameter & Description & Definition \\
\hline$\sigma$ & Initial vehicle utilization & $\sigma=0.85$ \\
\hline$\tau$ & Linear cooling factor & $\tau=0.3$ \\
\hline$i^{M A X}$ & Maximum number of total iterations & $i^{M A X}=\max \left\{200, n \cdot\left(m+m^{\prime}+m^{\prime \prime}+e\right) \cdot \varepsilon\right)$ \\
\hline$\varepsilon$ & Maximum iteration factor & \\
\hline$i^{W I}$ & Maximum number of iterations without new best known solution $\hat{s}$ & $i^{W I}=\max \left\{30, n \cdot\left(m+m^{\prime}+m^{\prime \prime}+e\right) \cdot \kappa\right\}$ \\
\hline$i^{E S-W I}$ & Number of iterations without new best solution after explicit shaking & $i^{E S-W I}=i^{W I}-1$ \\
\hline
\end{tabular}

The parameters listed in Table 8 are the result of comprehensive preliminary tests. With these parameter settings, best results can be achieved considering the trade-off between solution quality and computation time. The effects of the parameter $\varepsilon$ and the other parameters not listed in Table 8 are evaluated in terms of on solution quality and computation time in the next section.

\subsection{Performance of the VNS-variants}

The first part of this evaluation assesses the solution quality and computation time of the different VNS-variants at an aggregated level. Here, we consider all instance sets defined above (CHU, BOL, CE, G, CE-H, G-H, and N1 to N5) and compare the improvements in terms of computation time and solution quality (see Tables 9 and 10). All (mean) computation times resulting from experiments using one of the proposed VNS variants are performed on a computer with an Intel ${ }^{\circledR} \mathrm{XEON}^{\circledR} \mathrm{CPU}_{\mathrm{E} 5}$ at $2.9 \mathrm{GHz}$. Because realistic reference computation times are only available for the instance sets $\mathrm{CE}, \mathrm{G}, \mathrm{CE}-\mathrm{H}$, and $\mathrm{G}-\mathrm{H}$, the relative computation time improvements ("CT ${ }^{\text {Mean }}$ imp. [\%]") in Tables 9 and 10 are only based on the results for these sets. To assess the relative improvement of the solution quality, we use the minimum objective values obtained by our proposed solution methods to calculate the relative improvement "OV ${ }^{\mathrm{Min}}$ imp. [\%]". Reference values for comparison are the best known objective values either obtained by IBM CPLEX Optimizer 12.5 (after $50 \mathrm{~h}$ ) or taken from literature. The minimum objective values result from ten experiments per VNS-variant and parameter setting. In Tables 9 and 10, the ten experiment results for each instance and of all instance sets are aggregated to a single value. Tables 9 and 10 show these relative improvements for VNS, RVND, and VNSac with completely random vehicle selection $(\zeta=0$; Table 9$)$ and with distance proportionate vehicle selection $(\zeta=1$; Table 10$)$; and with respect to the parameters $i^{M A X}$ (maximum number of iterations), $\psi$ (improvement strategy), $\kappa$ (explicit shaking factor; zero means no explicit shaking), and $\theta$ (RD-transformation rule, whereby T ("true") means that the RD-transformation rule is available). Bold values in the tables mark the maximum improvements per column.

The numbers in Table 9 show that the FI-strategy is dominated by the BI-improvement strategy in terms of solution quality and that both explicit shaking mechanisms have a positive effect on the solution quality. The numbers also show that the RVND variant outperforms the other variants in terms of solution quality but at the expense of computation time. Regarding the maximum number of iterations, the effect of the parameter $\varepsilon$ on the trade-off between solution quality and computation time comes obvious. Best setting with respect to solution quality is RVND with $\zeta=0, \varepsilon=1, \psi=\mathrm{BI}, \kappa=0.1$, and $\theta=\mathrm{T}$, which achieves a mean improvement of $0.35 \%$ (and a computation time improvement of $18.0 \%$ ). Considering the best trade-off 
between solution quality and computation time, RVND with $\zeta=0, \varepsilon=0.5, \psi=\mathrm{BI}, \kappa=0.1$, and $\theta=\mathrm{F}$ performs best and achieves a mean solution quality improvement of $0.15 \%$ and a mean computation time reduction by $68.6 \%$.

The effect of the distance proportionate vehicle selection mechanism is shown in Table 10 . The numbers show that this mechanism is able to slightly improve the solution quality for almost all parameter settings with a similar computation time. For RVND with the first improvement strategy, computation times can be reduced remarkably. For $\zeta=1$, the best solution

Table 9

Relative improvements with $\zeta=0$ and with respect to the parameters $i^{M A X}, \psi, \kappa$, and $\theta$.

\begin{tabular}{|c|c|c|c|c|c|c|c|c|c|c|c|c|c|c|}
\hline \multirow{2}{*}{\multicolumn{3}{|c|}{$\varepsilon$}} & \multicolumn{4}{|l|}{ VNS } & \multicolumn{4}{|l|}{ RVND } & \multicolumn{4}{|l|}{ VNSac } \\
\hline & & & \multicolumn{2}{|l|}{1} & \multicolumn{2}{|l|}{0.5} & \multicolumn{2}{|l|}{1} & \multicolumn{2}{|l|}{0.5} & \multicolumn{2}{|l|}{1} & \multicolumn{2}{|l|}{0.5} \\
\hline$\psi$ & $\kappa$ & $\theta$ & $\begin{array}{l}\mathrm{OV}^{\mathrm{Min}} \\
\text { imp. [\%] }\end{array}$ & $\begin{array}{l}\mathrm{CT}^{\text {Mean }} \\
\text { imp. [\%] }\end{array}$ & $\begin{array}{l}\text { OV }^{\text {Min }} \\
\text { imp. [\%] }\end{array}$ & $\begin{array}{l}\mathrm{CT}^{\text {Mean }} \\
\text { imp. [\%] }\end{array}$ & $\begin{array}{l}\mathrm{OV}^{\mathrm{Min}} \\
\text { imp. [\%] }\end{array}$ & $\begin{array}{l}\mathrm{CT}^{\text {Mean }} \\
\text { imp. [\%] }\end{array}$ & $\begin{array}{l}\mathrm{OV}^{\mathrm{Min}} \\
\mathrm{imp} .[\%]\end{array}$ & $\begin{array}{l}\mathrm{CT}^{\text {Mean }} \\
\text { imp. [\%] }\end{array}$ & $\begin{array}{l}\mathrm{OV}^{\mathrm{Min}} \\
\text { imp. [\%] }\end{array}$ & $\begin{array}{l}\mathrm{CT}^{\text {Mean }} \\
\text { imp. [\%] }\end{array}$ & $\begin{array}{l}\mathrm{OV}^{\mathrm{Min}} \\
\text { imp. [\%] }\end{array}$ & $\begin{array}{l}\mathrm{CT}^{\text {Mean }} \\
\text { imp. [\%] }\end{array}$ \\
\hline FI & 0 & $\mathrm{~F}$ & -2.61 & 82.5 & -4.26 & 94.5 & -1.47 & 16.7 & -1.70 & 68.4 & -2.08 & 85.2 & -4.66 & 95.5 \\
\hline FI & 0.7 & $\mathrm{~F}$ & -2.48 & 82.6 & -3.21 & 94.2 & -1.17 & 16.3 & -1.69 & 68.5 & -2.06 & 85.0 & -4.36 & 95.8 \\
\hline FI & 0.3 & $\mathrm{~F}$ & -2.07 & 83.6 & -3.23 & 94.2 & -0.58 & 20.2 & -1.02 & 68.0 & -2.06 & 85.2 & -4.48 & 95.4 \\
\hline FI & 0.7 & $\mathrm{~T}$ & -2.26 & 83.4 & -3.51 & 94.2 & -1.09 & 16.6 & -1.67 & 69.0 & -2.55 & 84.3 & -4.22 & 95.2 \\
\hline FI & 0.3 & $\mathrm{~T}$ & -2.29 & 82.6 & -3.15 & 94.8 & -0.15 & 23.5 & -1.43 & 69.3 & -1.95 & 83.8 & -4.74 & 95.8 \\
\hline FI & 0.1 & $\mathrm{~T}$ & -1.81 & 82.8 & -3.33 & 94.2 & -0.19 & 35.7 & -0.40 & 67.8 & -2.15 & 84.0 & -5.42 & 95.9 \\
\hline \multicolumn{3}{|c|}{ Mean } & -2.19 & 83.0 & -3.45 & 94.4 & -0.67 & 21.9 & -1.19 & 68.4 & -2.18 & 84.7 & -4.62 & 95.6 \\
\hline BI & 0 & $\mathrm{~F}$ & -0.63 & 48.6 & -1.43 & 79.9 & -1.36 & 9.8 & -1.42 & 69.0 & -0.31 & 51.6 & -1.08 & 76.0 \\
\hline BI & 0.7 & $\mathrm{~F}$ & -0.84 & 49.2 & -0.98 & 79.9 & -0.52 & 9.7 & -0.12 & 69.2 & -0.27 & 51.2 & -0.98 & 76.5 \\
\hline BI & 0.3 & $\mathrm{~F}$ & -0.25 & 50.0 & -0.99 & 79.9 & -0.03 & 12.1 & 0.14 & 69.6 & -0.20 & 51.6 & -1.16 & 75.7 \\
\hline BI & 0.7 & $\mathrm{~T}$ & -0.80 & 50.5 & -1.13 & 79.8 & -0.62 & 11.0 & -0.07 & 68.5 & -0.25 & 49.9 & -1.18 & 77.0 \\
\hline BI & 0.3 & $\mathrm{~T}$ & -0.32 & 49.4 & -0.77 & 79.8 & -0.06 & 13.2 & -0.05 & 69.7 & -0.18 & 50.9 & -1.01 & 76.2 \\
\hline BI & 0.1 & $\mathrm{~T}$ & -0.39 & 48.3 & -1.13 & 79.8 & 0.35 & 18.0 & -0.02 & 73.6 & -0.35 & 51.5 & -1.41 & 75.7 \\
\hline \multicolumn{3}{|c|}{ Mean } & -0.50 & 49.2 & -1.03 & 79.9 & -0.31 & 12.4 & -0.20 & 69.7 & -0.22 & 51.2 & -1.14 & 76.1 \\
\hline
\end{tabular}

method and parameter setting for $\varepsilon=1$ is defined by RVND and $\psi=\mathrm{BI}, \kappa=0.3$, and $\theta=\mathrm{F}$; here, a mean improvement of $0.19 \%$ (with a computation time improvement of $11.7 \%$ ) is achieved. For $\zeta=1$ and $\varepsilon=0.5$, RVND with $\psi=\mathrm{BI}, \kappa=0.3$, and $\theta=T$ performs best and improves solution quality by $0.20 \%$ and reduces mean computation time by $69.8 \%$.

Summarizing this first analysis, we conclude that the RVND-variant with $\zeta=0, \varepsilon=1, \psi=\mathrm{BI}, \kappa=0.1$, and $\theta=\mathrm{T}\left(\mathrm{RVND}^{\mathrm{SQ}}\right)$ performs best in terms of solution quality and that RVND with $\zeta=1, \varepsilon=0.5, \psi=\mathrm{BI}, \kappa=0.3$, and $\theta=\mathrm{T}\left(\mathrm{RVND}^{\mathrm{TO}}\right)$ performs best regarding the trade-off between solution quality and computation time. Of course, this trade-off always depends on the decision maker's preferences.

In the second part of this evaluation, we compare the objective values achieved by RVND ${ }^{\mathrm{SQ}}$ and RVND ${ }^{\mathrm{TO}}$ with the reference values of the instance sets $\mathrm{CE}, \mathrm{G}, \mathrm{CE}-\mathrm{H}$, and $\mathrm{G}-\mathrm{H}$ to assess the solution quality in a more detailed way. Therefore, for each instance in these sets, Tables 11 and 12 list the solution method ("SM"; for abbreviations, see Section 1) the best known

Table 10

Relative improvements with $\zeta=1$ and with respect to the parameters $i^{M A X}, \psi, \kappa$, and $\theta$

\begin{tabular}{|c|c|c|c|c|c|c|c|c|c|c|c|c|c|c|}
\hline \multirow{2}{*}{\multicolumn{3}{|c|}{$i^{\operatorname{MAX}}$}} & \multicolumn{4}{|l|}{ VNS } & \multicolumn{4}{|l|}{ RVND } & \multicolumn{4}{|l|}{ VNSac } \\
\hline & & & \multicolumn{2}{|l|}{1} & \multicolumn{2}{|l|}{0.5} & \multicolumn{2}{|l|}{1} & \multicolumn{2}{|l|}{0.5} & \multicolumn{2}{|l|}{1} & \multicolumn{2}{|l|}{0.5} \\
\hline$\psi$ & $\kappa$ & $\theta$ & $\begin{array}{l}\mathrm{OV}^{\mathrm{Min}} \\
\text { imp. [\%] }\end{array}$ & $\begin{array}{l}\mathrm{CT}^{\text {Mean }} \\
\text { imp. [\%] }\end{array}$ & $\begin{array}{l}\mathrm{OV}^{\mathrm{Min}} \\
\text { imp. [\%] }\end{array}$ & $\begin{array}{l}\mathrm{CT}^{\text {Mean }} \\
\text { imp. [\%] }\end{array}$ & $\begin{array}{l}\mathrm{OV}^{\mathrm{Min}} \\
\text { imp. [\%] }\end{array}$ & $\begin{array}{l}\mathrm{CT}^{\text {Mean }} \\
\text { imp. [\%] }\end{array}$ & $\begin{array}{l}\mathrm{OV}^{\mathrm{Min}} \\
\text { imp. [\%] }\end{array}$ & $\begin{array}{l}\mathrm{CT}^{\text {Mean }} \\
\text { imp. [\%] }\end{array}$ & $\begin{array}{l}\mathrm{OV}^{\mathrm{Min}} \\
\text { imp. [\%] }\end{array}$ & $\begin{array}{l}\mathrm{CT}^{\text {Mean }} \\
\text { imp. [\%] }\end{array}$ & $\begin{array}{l}\mathrm{OV}^{\mathrm{Min}} \\
\text { imp. [\%] }\end{array}$ & $\begin{array}{l}\mathrm{CT}^{\text {Mean }} \\
\text { imp. [\%] }\end{array}$ \\
\hline FI & 0 & $\mathrm{~F}$ & -1.87 & 82.7 & -3.95 & 94.8 & -1.64 & 46.9 & -1.65 & 67.9 & -2.04 & 86.3 & -4.74 & 95.6 \\
\hline FI & 0.7 & $\mathrm{~F}$ & -2.53 & 82.6 & -3.66 & 94.0 & -1.10 & 45.5 & -1.66 & 70.1 & -2.10 & 86.1 & -4.29 & 95.9 \\
\hline FI & 0.3 & $\mathrm{~F}$ & -2.21 & 82.3 & -3.25 & 94.1 & -0.37 & 47.9 & -0.65 & 68.5 & -1.78 & 86.4 & -4.51 & 95.8 \\
\hline FI & 0.7 & $\mathrm{~T}$ & -2.33 & 82.5 & -3.20 & 94.1 & -1.19 & 46.6 & -1.50 & 68.2 & -2.30 & 85.4 & -4.79 & 95.6 \\
\hline FI & 0.3 & $\mathrm{~T}$ & -1.83 & 82.9 & -3.23 & 94.0 & -0.36 & 49.9 & -0.81 & 69.3 & -2.12 & 86.6 & -4.52 & 95.9 \\
\hline FI & 0.1 & $\mathrm{~T}$ & -1.76 & 82.2 & -3.28 & 94.0 & -0.37 & 54.8 & -0.29 & 67.6 & -1.99 & 86.2 & -4.93 & 95.8 \\
\hline \multicolumn{2}{|c|}{ Mean } & & -1.99 & 82.5 & -3.37 & 94.3 & -0.72 & 48.7 & -0.98 & 68.5 & -2.01 & 86.2 & -4.65 & 95.8 \\
\hline BI & 0 & $\mathrm{~F}$ & -0.62 & 50.2 & -1.45 & 79.8 & -1.33 & 10.4 & -1.39 & 69.2 & -0.35 & 51.5 & -0.96 & 78.2 \\
\hline BI & 0.7 & $\mathrm{~F}$ & -0.45 & 47.9 & -0.97 & 80.0 & -0.30 & 10.1 & 0.18 & 69.3 & -0.40 & 58.2 & -1.30 & 78.2 \\
\hline BI & 0.3 & $\mathrm{~F}$ & 0.02 & 49.7 & -0.67 & 80.2 & 0.19 & 11.7 & 0.16 & 69.7 & -0.25 & 58.3 & -1.02 & 78.3 \\
\hline BI & 0.7 & $\mathrm{~T}$ & -0.56 & 57.5 & -1.02 & 77.0 & -0.48 & 11.7 & -0.03 & 69.4 & -0.26 & 58.2 & -0.98 & 78.2 \\
\hline BI & 0.3 & $\mathrm{~T}$ & 0.16 & 49.0 & -0.99 & 79.9 & 0.08 & 12.9 & 0.20 & 69.8 & -0.39 & 58.1 & -1.06 & 78.3 \\
\hline BI & 0.1 & $\mathrm{~T}$ & -0.13 & 48.4 & -0.80 & 79.9 & -0.04 & 18.4 & 0.10 & 69.7 & -0.21 & 58.6 & -1.27 & 78.2 \\
\hline \multicolumn{2}{|c|}{ Mean } & & -0.28 & 50.1 & -0.99 & 79.5 & -0.25 & 12.7 & -0.10 & 69.4 & -0.30 & 56.3 & -1.13 & 78.2 \\
\hline
\end{tabular}


objective value ("BKOV") is achieved with and the mean computation time of the BKOV ("CT") in seconds. For both parameter settings ( $\mathrm{RVND}^{\mathrm{SQ}}$ and $\mathrm{RVND}^{\mathrm{TO}}$ ), the minimum objective value ("OV") from the ten calculated solutions, the corresponding relative improvement concerning BKOV and OV ("Imp. OV"), and the mean relative computation time improvement ("Imp. CT"), are presented. In both tables, improvements of the BKOV are marked bold. Because no detailed computation times for MS-LI, MS-ILS, and UGHS are given in Vidal et al. (2015), their results are not used for comparisons in Table 11 (a comparison between RVND ${ }^{\mathrm{SQ}}, \mathrm{RVND}^{\mathrm{TO}}$, and all other solution methods is provided by Tables 16-19 in Appendix C).

The results of Tables 11 and 12 show the general suitability of RVND ${ }^{S Q}$ and RVND ${ }^{\mathrm{TO}}$ to solve VRPPCs. However, it has to be remarked that the effectiveness of RVND, particularly designed for the VRPPCdR, is inferior compared to the other solution methods developed for the VRPPC. Nevertheless, for the three instances G-19, G-H-04, and G-H-19, improvements of the best known objective values are achieved.

Table 11

Results of RVND ${ }^{\mathrm{SQ}}$ and RVND ${ }^{\mathrm{TO}}$ on instance sets CE and G.

\begin{tabular}{|c|c|c|c|c|c|c|c|c|c|}
\hline \multirow[t]{2}{*}{ Instance } & \multirow[t]{2}{*}{ SM } & \multirow[t]{2}{*}{ BKOV } & \multirow[t]{2}{*}{$\mathrm{CT}[\mathrm{s}]$} & \multicolumn{3}{|l|}{$\mathrm{RVND}^{\mathrm{SQ}}$} & \multicolumn{3}{|l|}{ RVND $^{\mathrm{TO}}$} \\
\hline & & & & OV & Imp. OV [\%] & Imp. CT [\%] & OV & Imp. OV [\%] & Imp. CT [\%] \\
\hline CE-01 & TS & 1119.47 & 24.3 & 1135.81 & -1.46 & 87.1 & 1126.24 & -0.60 & 94.0 \\
\hline CE-02 & TS & 1814.52 & 33.0 & 1841.69 & -1.50 & 79.9 & 1835.45 & -1.15 & 95.9 \\
\hline CE-03 & AVNS & 1920.86 & 212.1 & 1965.90 & -2.34 & 88.9 & 1967.35 & -2.42 & 96.6 \\
\hline CE-04 & AVNS & 2512.05 & 279.7 & 2564.22 & -2.08 & 79.5 & 2565.11 & -2.11 & 94.1 \\
\hline CE-05 & AVNS & 3099.77 & 228.6 & 3162.54 & -2.03 & 60.8 & 3171.29 & -2.31 & 88.8 \\
\hline CE-06 & TS+ & 1207.47 & 25.2 & 1209.62 & -0.18 & 85.9 & 1220.91 & -1.11 & 94.4 \\
\hline CE-07 & TS & 2006.52 & 32.7 & 2049.29 & -2.13 & 84.4 & 2051.09 & -2.22 & 95.7 \\
\hline CE-08 & AVNS & 2052.05 & 253.1 & 2087.58 & -1.73 & 91.3 & 2087.62 & -1.73 & 97.2 \\
\hline CE-09 & AVNS & 2432.51 & 259.0 & 2451.90 & -0.80 & 79.1 & 2454.59 & -0.91 & 94.1 \\
\hline CE-10 & AVNS & 3391.35 & 201.0 & 3445.89 & -1.61 & 54.9 & 3459.41 & -2.01 & 87.2 \\
\hline CE-11 & AVNS & 2332.21 & 316.0 & 2338.83 & -0.28 & 83.1 & 2339.11 & -0.30 & 94.9 \\
\hline CE-12 & TS+ & 1952.86 & 59.5 & 1970.87 & -0.92 & 71.8 & 1956.71 & -0.20 & 92.1 \\
\hline CE-13 & AVNS & 2858.94 & 278.5 & 2864.16 & -0.18 & 81.0 & 2868.93 & -0.35 & 94.1 \\
\hline CE-14 & AVNS & 2215.38 & 93.2 & 2224.25 & -0.40 & 81.9 & 2234.57 & -0.87 & 94.7 \\
\hline Mean CE & & & & & -1.26 & 79.3 & & -1.31 & 93.8 \\
\hline G-01 & AVNS & $14,157.08$ & 652.6 & $14,283.09$ & -0.89 & -5.6 & $14,295.11$ & -0.97 & 47.9 \\
\hline G-02 & AVNS & $19,204.36$ & 1558.4 & $19,394.37$ & -0.99 & -52.2 & $19,436.23$ & -1.21 & 25.1 \\
\hline G-03 & TS+ & $24,592.18$ & 5940.4 & $24,824.57$ & -0.94 & -28.9 & $24,834.50$ & -0.99 & 36.8 \\
\hline G-04 & AVNS & $34,415.82$ & 2500.9 & $34,793.08$ & -1.10 & -520.7 & $34,841.65$ & -1.24 & -168.5 \\
\hline G-05 & TS+ & $14,261.31$ & 847.8 & $14,489.59$ & -1.60 & -57.2 & $14,572.95$ & -2.19 & 36.5 \\
\hline G-06 & AVNS & $21,440.79$ & 1783.5 & $21,684.74$ & -1.14 & -79.2 & $21,820.27$ & -1.77 & 30.5 \\
\hline G-07 & AVNS & $23,375.60$ & 2262.8 & $23,767.21$ & -1.68 & -168.1 & $23,739.21$ & -1.56 & -2.6 \\
\hline G-08 & AVNS & $29,797.62$ & 2339.7 & $30,079.61$ & -0.95 & -301.2 & $30,082.38$ & -0.96 & -58.2 \\
\hline G-09 & TS+ & 1325.62 & 819.1 & 1358.61 & -2.49 & 33.0 & 1354.38 & -2.17 & 76.3 \\
\hline G-10 & TS+ & 1590.82 & 1762.3 & 1621.51 & -1.93 & 34.5 & 1633.94 & -2.71 & 76.5 \\
\hline G-11 & TS & 2172.28 & 1492.7 & 2218.96 & -2.15 & -67.6 & 2222.61 & -2.32 & 38.5 \\
\hline G-12 & TS & 2492.75 & 2309.7 & 2549.76 & -2.29 & -117.0 & 2540.85 & -1.93 & 21.0 \\
\hline G-13 & TS+ & 2274.12 & 504.5 & 2326.12 & -2.29 & 78.9 & 2326.26 & -2.29 & 92.9 \\
\hline G-14 & TS+ & 2703.31 & 976.9 & 2792.45 & -3.30 & 75.5 & 2802.60 & -3.67 & 91.6 \\
\hline G-15 & TS & 3158.92 & 924.8 & 3270.84 & -3.54 & 49.4 & 3280.20 & -3.84 & 82.8 \\
\hline G-16 & TS+ & 3638.39 & 4675.1 & 3779.11 & -3.87 & 81.4 & 3772.98 & -3.70 & 93.6 \\
\hline G-17 & TS+ & 1633.35 & 472.0 & 1658.32 & -1.53 & 76.4 & 1657.05 & -1.45 & 92.2 \\
\hline G-18 & TS & 2705.90 & 622.0 & 2708.61 & -0.10 & 70.7 & 2729.05 & -0.86 & 90.6 \\
\hline G-19 & TS & 3497.54 & 1012.5 & 3473.82 & 0.68 & 73.2 & 3501.38 & -0.11 & 91.1 \\
\hline G-20 & TS+ & 4306.89 & 2476.3 & 4321.60 & -0.34 & 85.2 & 4335.79 & -0.67 & 95.1 \\
\hline Mean G & & & & & -1.62 & -37.0 & & -1.83 & 44.5 \\
\hline Mean & & & & & -1.47 & 10.9 & & -1.61 & 64.8 \\
\hline
\end{tabular}

Table 12

Results of RVND ${ }^{\mathrm{SQ}}$ and RVND ${ }^{\mathrm{TO}}$ on the instance sets CE-H and G-H.

\begin{tabular}{|c|c|c|c|c|c|c|c|c|c|}
\hline \multirow[t]{2}{*}{ Instance } & \multirow[t]{2}{*}{ SM } & \multirow[t]{2}{*}{ BKOV } & \multirow[t]{2}{*}{$\mathrm{CT}[\mathrm{s}]$} & \multicolumn{3}{|l|}{$\mathrm{RVND}^{\mathrm{SQ}}$} & \multicolumn{3}{|l|}{$\mathrm{RVND}^{\mathrm{TO}}$} \\
\hline & & & & OV & Imp. OV [\%] & Imp. CT [\%] & OV & Imp. OV [\%] & Imp. CT [\%] \\
\hline CE-H-01 & TS & 1191.70 & 25.7 & 1210.28 & -1.56 & 86.7 & 1199.03 & -0.61 & 93.8 \\
\hline CE-H-02 & TS+ & 1791.21 & 34.8 & 1828.29 & -2.07 & 85.1 & 1834.25 & -2.40 & 95.6 \\
\hline CE-H-03 & TS+ & 1917.96 & 80.8 & 1944.63 & -1.39 & 70.8 & 1936.65 & -0.97 & 91.1 \\
\hline CE-H-04 & TS & 2481.64 & 195.6 & 2539.38 & -2.33 & 68.5 & 2500.89 & -0.78 & 92.3 \\
\hline CE-H-05 & TS+ & 3143.01 & 342.4 & 3219.14 & -2.42 & 76.9 & 3195.91 & -1.68 & 94.6 \\
\hline CE-H-06 & TS+ & 1206.82 & 25.1 & 1218.85 & -1.00 & 87.0 & 1220.39 & -1.12 & 95.3 \\
\hline CE-H-07 & TS+ & 2031.85 & 32.0 & 2053.31 & -1.06 & 85.2 & 2061.65 & -1.47 & 96.4 \\
\hline CE-H-08 & TS+ & 1986.51 & 84.5 & 2024.93 & -1.93 & 66.7 & 2024.96 & -1.94 & 92.0 \\
\hline
\end{tabular}


Table 12 (continued)

\begin{tabular}{|c|c|c|c|c|c|c|c|c|c|}
\hline \multirow[t]{2}{*}{ Instance } & \multirow[t]{2}{*}{ SM } & \multirow[t]{2}{*}{ BKOV } & \multirow[t]{2}{*}{$\mathrm{CT}[\mathrm{s}]$} & \multicolumn{3}{|l|}{$\mathrm{RVND}^{\mathrm{SQ}}$} & \multicolumn{3}{|l|}{$\mathrm{RVND}^{\mathrm{TO}}$} \\
\hline & & & & OV & Imp. OV [\%] & Imp. CT [\%] & OV & Imp. OV [\%] & Imp. CT [\%] \\
\hline CE-H-09 & TS & 2445.49 & 188.9 & 2502.81 & -2.34 & 71.2 & 2497.76 & -2.14 & 93.2 \\
\hline CE-H-10 & TS & 3271.70 & 309.5 & 3304.86 & -1.01 & 66.9 & 3331.62 & -1.83 & 92.3 \\
\hline CE-H-11 & RIP & 2308.76 & 18.8 & 2323.08 & -0.62 & -183.3 & 2331.63 & -0.99 & 30.5 \\
\hline CE-H-12 & RIP & 1908.74 & 13.0 & 1920.32 & -0.61 & -30.8 & 1918.97 & -0.54 & 70.1 \\
\hline CE-H-13 & RIP & 2842.18 & 19.5 & 2855.39 & -0.46 & -156.6 & 2882.67 & -1.42 & 33.7 \\
\hline CE-H-14 & TS+ & 1907.75 & 67.2 & 1914.08 & -0.33 & 71.1 & 1917.14 & -0.49 & 93.1 \\
\hline Mean CE-H & & & & & -1.37 & 33.3 & & -1.31 & 83.1 \\
\hline G-H-01 & TS & $14,174.27$ & 642.5 & $14,191.65$ & -0.12 & -17.5 & $14,197.28$ & -0.16 & 55.6 \\
\hline $\mathrm{G}-\mathrm{H}-02$ & TS+ & $18,537.70$ & 4955.3 & $18,541.59$ & -0.02 & 41.2 & $18,571.03$ & -0.18 & 78.0 \\
\hline $\mathrm{G}-\mathrm{H}-03$ & TS+ & $25,177.92$ & $11,996.2$ & $25,297.58$ & -0.48 & 39.4 & $25,306.09$ & -0.51 & 75.6 \\
\hline $\mathrm{G}-\mathrm{H}-04$ & TS+ & $34,991.21$ & 4079.1 & $34,794.70$ & 0.56 & -284.4 & $34,747.39$ & 0.70 & -55.7 \\
\hline G-H-05 & TS+ & $15,411.82$ & 754.2 & $15,714.24$ & -1.96 & -50.1 & $15,751.18$ & -2.20 & 41.1 \\
\hline G-H-06 & TS+ & $19,859.30$ & 1954.1 & $20,116.24$ & -1.29 & -53.4 & $20,204.73$ & -1.74 & 41.2 \\
\hline G-H-07 & TS+ & $23,481.28$ & 9167.3 & $23,751.16$ & -1.15 & 33.3 & $23,716.72$ & -1.00 & 72.4 \\
\hline $\mathrm{G}-\mathrm{H}-08$ & TS+ & $27,334.84$ & $18,625.2$ & $27,482.48$ & -0.54 & 36.0 & $27,433.18$ & -0.36 & 75.1 \\
\hline G-H-09 & TS+ & 1329.27 & 1829.3 & 1373.90 & -3.36 & 74.2 & 1371.14 & -3.15 & 90.9 \\
\hline G-H-10 & TS & 1554.96 & 1087.1 & 1611.09 & -3.61 & -13.4 & 1604.15 & -3.16 & 55.0 \\
\hline G-H-11 & TS & 2191.23 & 1445.5 & 2246.13 & -2.51 & -38.4 & 2244.60 & -2.44 & 45.9 \\
\hline $\mathrm{G}-\mathrm{H}-12$ & TS+ & 2482.92 & 4224.0 & 2554.43 & -2.88 & -7.2 & 2559.52 & -3.09 & 60.0 \\
\hline G-H-13 & TS & 2231.88 & 405.8 & 2307.28 & -3.38 & 75.0 & 2300.13 & -3.06 & 91.4 \\
\hline $\mathrm{G}-\mathrm{H}-14$ & TS+ & 2684.70 & 1042.9 & 2752.90 & -2.54 & 78.8 & 2767.00 & -3.07 & 92.8 \\
\hline G-H-15 & TS & 3123.60 & 976.6 & 3222.33 & -3.16 & 52.6 & 3237.13 & -3.63 & 84.5 \\
\hline G-H-16 & TS+ & 3621.85 & 6217.4 & 3768.94 & -4.06 & 87.7 & 3770.16 & -4.09 & 95.9 \\
\hline G-H-17 & TS+ & 1664.08 & 522.6 & 1680.94 & -1.01 & 80.8 & 1685.81 & -1.31 & 93.7 \\
\hline G-H-18 & TS+ & 2708.73 & 1132.5 & 2730.16 & -0.79 & 86.5 & 2727.52 & -0.69 & 95.6 \\
\hline G-H-19 & TS+ & 3443.59 & 1627.1 & 3439.96 & 0.11 & 86.4 & 3449.35 & -0.17 & 95.3 \\
\hline $\mathrm{G}-\mathrm{H}-20$ & TS & 4306.53 & 1392.2 & 4312.65 & -0.14 & 80.2 & 4334.54 & -0.65 & 93.0 \\
\hline Mean G-H & & & & & -1.62 & 19.4 & & -1.70 & 68.9 \\
\hline Mean & & & & & -1.51 & 25.1 & & -1.54 & 74.7 \\
\hline
\end{tabular}

Regarding the trade-off between solution quality and computation time, it has to be emphasized that RVND ${ }^{\mathrm{TO}}$ has a remarkable efficiency for almost every instance compared to the other solution methods: RVND ${ }^{\text {TO }}$ achieves for homogeneous and heterogeneous vehicle fleets small mean degradations of $1.61 \%$ and $1.54 \%$, whereby computation times are reduced by $64.8 \%$ and $74.7 \%$.

\subsection{Economic benefits and implications of solving the VRPPCdR}

Purpose of this part of the analysis is the assessment of the economic benefits (cost reductions) achieved by solving the VRPPCdR and the investigation of further managerial implications. Therefore, we compare the costs between the two planning approaches VRPPCdR and VRPPC with regard to the basic data scenarios provided by the BOL instance set. Table 13 first lists the objective value ("OV"), the relative gap reported by CPLEX ("Rel. Gap"), and the "used vehicles" for the solutions calculated by CPLEX 12.5 (terminated after $50 \mathrm{~h}$ ) for the VRPPCdR instance sets N1 to N5. Next columns contain the objective value ("OVVVND-SQ"), the relative improvement of RVND ${ }^{\mathrm{SQ}}$ comparing $\mathrm{OV}^{\mathrm{RVND}-\mathrm{SQ}}$ and $\mathrm{OV}^{\mathrm{C}}$ ("Rel. Imp. OV"), and the list of "used vehicles" for the best solution (out of ten) calculated by RVND ${ }^{S Q}$. The column "Identical" compares the list of used vehicles and the calculated delivery costs:

- Used vehicles and cost are identical: $\sqrt{ }$

- Used vehicles are identical and the costs achieved by RVND ${ }^{\text {SQ }}$ are lower:+

- Used vehicles are not identical and the costs achieved by RVND ${ }^{\mathrm{SQ}}$ are lower: ++

- Used vehicles are identical and the costs of RVND ${ }^{S Q}$ are higher: -

- Used vehicles are not identical and the costs of RVND ${ }^{\mathrm{SQ}}$ are higher: --

The last two columns compare the total cost ("TC") improvements of the RVND ${ }^{\text {SQ }}$ solutions of the VRPPCdR instance with the total costs ("TC $\mathrm{BOL}_{-1}, \ldots, 5$ "; including fixed costs) of the original VRPPC instance (see first column or Table 14 in Appendix (). The column "used vehicles" shows that the private fleet is utilized before any other delivery mode is applied. This is in line with the assumption that FTL deliveries with the private fleet are more cost-efficient than using other delivery modes. Concerning this, we conclude that the parameters of the rental options are reasonable.

Comparing the solutions calculated by CPLEX and $\mathrm{RVND}^{\mathrm{SQ}}, \mathrm{RVND}^{\mathrm{SQ}}$ selects almost for each problem instance the most suitable delivery modes (or even better ones) and makes only in three cases (N3-04, N3-05, and N3-06) another mode selection decision than CPLEX leading to higher costs. Based on this observation, it can be stated that the RVND ${ }^{S Q}$ solution method is able to determine 
Table 13

Results of RVND ${ }^{\mathrm{SQ}}$ on instance sets $\mathrm{N} 1$ to N5

\begin{tabular}{|c|c|c|c|c|c|c|c|c|c|c|c|c|}
\hline \multicolumn{2}{|c|}{ Instance } & \multicolumn{3}{|c|}{ CPLEX 12.5 (after $50 \mathrm{~h}$ ) } & \multicolumn{8}{|l|}{$\mathrm{RVND}^{\mathrm{SQ}}$} \\
\hline & & $\mathrm{OV}^{\mathrm{C}}$ & $\begin{array}{l}\text { Rel. } \\
\text { Gap [\%] }\end{array}$ & $\begin{array}{l}\text { Used } \\
\text { vehicles }\end{array}$ & $\mathrm{OV}^{\mathrm{RVND}-S \mathrm{Q}}$ & $\begin{array}{l}\text { Rel. Imp. } \\
\text { OV [\%] }\end{array}$ & $\mathrm{CT}[\mathrm{s}]$ & $\begin{array}{l}\text { Used } \\
\text { vehicles }\end{array}$ & Identical & $\mathrm{TC}_{\mathrm{BOL}-1, \ldots, 5}$ & TC & $\begin{array}{l}\text { Rel. Imp. } \\
\text { TC [\%] }\end{array}$ \\
\hline N1 & $\begin{array}{l}-01 \\
-02 \\
-03 \\
-04 \\
-05 \\
-06 \\
-07\end{array}$ & $\begin{array}{l}333.76 \\
332.64 \\
234.00 \\
234.00 \\
234.00 \\
234.00 \\
234.00\end{array}$ & $\begin{array}{l}0.0 \\
0.0 \\
0.0 \\
0.0 \\
0.0 \\
0.0 \\
0.0\end{array}$ & $\begin{array}{l}\mathrm{V} 1 ; \mathrm{C} 2 \\
\mathrm{~V} 1 ; \mathrm{C} 1 ; \mathrm{C} 2 \\
\mathrm{~V} 1 ; \mathrm{D} 2 \\
\mathrm{~V} 1 ; \mathrm{D} 2 \\
\mathrm{~V} 1 ; \mathrm{D} 2 \\
\mathrm{~V} 1 ; \mathrm{D} 2 \\
\mathrm{~V} 1 ; \mathrm{D} 2\end{array}$ & $\begin{array}{l}333.76 \\
332.64 \\
234.00 \\
234.00 \\
234.00 \\
234.00 \\
234.00\end{array}$ & $\begin{array}{l}0.0 \\
0.0 \\
0.0 \\
0.0 \\
0.0 \\
0.0 \\
0.0\end{array}$ & $\begin{array}{l}0.2 \\
0.4 \\
0.4 \\
0.6 \\
0.7 \\
0.6 \\
0.7\end{array}$ & $\begin{array}{l}\mathrm{V} 1 ; \mathrm{C} 2 \\
\mathrm{~V} 1 ; \mathrm{C} 1 ; \mathrm{C} 2 \\
\mathrm{~V} 1 ; \mathrm{D} 2 \\
\mathrm{~V} 1 ; \mathrm{D} 2 \\
\mathrm{~V} 1 ; \mathrm{D} 2 \\
\mathrm{~V} 1 ; \mathrm{D} 2 \\
\mathrm{~V} 1 ; \mathrm{D} 2\end{array}$ & $\begin{array}{l}\sqrt{ } \\
\sqrt{ } \\
\sqrt{ } \\
\sqrt{ } \\
\sqrt{ } \\
\sqrt{ } \\
\sqrt{ }\end{array}$ & $\mathrm{TC}_{\mathrm{BOL}-1}=423.5$ & $\begin{array}{l}393.76 \\
392.64 \\
294.00 \\
294.00 \\
294.00 \\
294.00 \\
294.00\end{array}$ & $\begin{array}{l}7.0 \\
7.3 \\
30.6 \\
30.6 \\
30.6 \\
30.6 \\
30.6\end{array}$ \\
\hline $\mathrm{N} 2$ & $\begin{array}{l}-01 \\
-02 \\
-03 \\
-04 \\
-05 \\
-06 \\
-07\end{array}$ & $\begin{array}{l}431.52 \\
385.52 \\
298.50 \\
293.00 \\
304.54 \\
372.97 \\
293.00\end{array}$ & $\begin{array}{l}0.0 \\
0.0 \\
0.0 \\
0.0 \\
0.0 \\
0.0 \\
0.0\end{array}$ & $\begin{array}{l}\text { V2;C2;C3 } \\
\text { V2;C4 } \\
\text { V2;M1 } \\
\text { V2;M2;C1 } \\
\text { V2;M2;C4 } \\
\text { V2;D2;C3 } \\
\text { V2;M2;C1 }\end{array}$ & $\begin{array}{l}431.52 \\
385.52 \\
298.50 \\
293.00 \\
304.54 \\
372.97 \\
293.00\end{array}$ & $\begin{array}{l}0.0 \\
0.0 \\
0.0 \\
0.0 \\
0.0 \\
0.0 \\
0.0\end{array}$ & $\begin{array}{l}0.2 \\
0.6 \\
1.3 \\
1.3 \\
1.0 \\
1.3 \\
2.0\end{array}$ & $\begin{array}{l}\mathrm{V} 2 ; \mathrm{C} 2 ; \mathrm{C} 3 \\
\mathrm{~V} 2 ; \mathrm{C} 4 \\
\mathrm{~V} 2 ; \mathrm{M} 1 \\
\mathrm{~V} 2 ; \mathrm{M} 2 ; \mathrm{C} 1 \\
\mathrm{~V} 2 ; \mathrm{M} 2 ; \mathrm{C} 4 \\
\mathrm{~V} 2 ; \mathrm{D} 2 ; \mathrm{C} 3 \\
\mathrm{~V} 2 ; \mathrm{M} 2 ; \mathrm{C} 1\end{array}$ & $\begin{array}{l}\sqrt{ } \\
\sqrt{ } \\
\sqrt{ } \\
\sqrt{ } \\
\sqrt{ } \\
\sqrt{ } \\
\sqrt{ }\end{array}$ & $\mathrm{TC}_{\mathrm{BOL}-2}=476.5$ & $\begin{array}{l}551.52 \\
505.52 \\
418.50 \\
413.00 \\
424.54 \\
492.97 \\
413.00\end{array}$ & $\begin{array}{l}-15.7 \\
-6.1 \\
12.2 \\
13.3 \\
10.9 \\
-3.5 \\
13.3\end{array}$ \\
\hline N3 & $\begin{array}{l}-01 \\
-02 \\
-03 \\
-04 \\
-05 \\
-06 \\
-07\end{array}$ & $\begin{array}{l}501.49 \\
456.00 \\
393.00 \\
386.25 \\
386.25 \\
490.50 \\
385.25\end{array}$ & $\begin{array}{l}25.3 \\
20.4 \\
37.4 \\
0.0 \\
0.0 \\
57.8 \\
31.1\end{array}$ & $\begin{array}{l}\text { V3;V4;C3 } \\
\text { V3;V4;C1 } \\
\text { V3;V4;M1 } \\
\text { V3;V4;M2 } \\
\text { V3;V4;M2 } \\
\text { V3;V4;D2 } \\
\text { V3;V4;M2 }\end{array}$ & $\begin{array}{l}501.49 \\
456.00 \\
385.50 \\
388.50 \\
399.10 \\
507.02 \\
385.00\end{array}$ & $\begin{array}{l}0.0 \\
0.0 \\
1.9 \\
-0.6 \\
-3.3 \\
-3.4 \\
0.3\end{array}$ & $\begin{array}{l}0.8 \\
1.5 \\
1.7 \\
1.7 \\
1.3 \\
2.2 \\
0.7\end{array}$ & $\begin{array}{l}\text { V3;V4;C3 } \\
\text { V3;V4;C1 } \\
\text { V3;V4;M1 } \\
\text { V3;V4;M2;C1 } \\
\text { V3;V4;M2;C4 } \\
\text { V3;V4;C3 } \\
\text { V3;V4;M1 }\end{array}$ & $\begin{array}{l}\sqrt{ } \\
\sqrt{ } \\
+ \\
-- \\
-- \\
-- \\
++\end{array}$ & $\mathrm{TC}_{\mathrm{BOL}-3}=777.0$ & $\begin{array}{l}791.49 \\
746.00 \\
675.00 \\
685.25 \\
650.00 \\
791.49 \\
675.50\end{array}$ & $\begin{array}{l}-1.9 \\
4.0 \\
13.1 \\
11.8 \\
16.3 \\
-1.9 \\
13.1\end{array}$ \\
\hline N4 & $\begin{array}{l}-01 \\
-02 \\
-03 \\
-04 \\
-05 \\
-06 \\
-07\end{array}$ & $\begin{array}{l}1802.75 \\
1278.00 \\
1056.00 \\
1112.50 \\
1385.75 \\
1400.00 \\
1043.50\end{array}$ & $\begin{array}{l}48.1 \\
26.3 \\
43.9 \\
26.3 \\
35.7 \\
59.3 \\
44.4\end{array}$ & $\begin{array}{l}\text { V5;C2;C3 } \\
\text { V5;C1;C3 } \\
\text { V5;M1 } \\
\text { V5;M3;C1 } \\
\text { V5;M2;D2 } \\
\text { V5;M3;D2 } \\
\text { V5;M1;M3 }\end{array}$ & $\begin{array}{l}1339.24 \\
1278.00 \\
1043.50 \\
1082.50 \\
1185.14 \\
1171.14 \\
1043.50\end{array}$ & $\begin{array}{l}25.7 \\
0.0 \\
1.2 \\
2.7 \\
14.5 \\
16.3 \\
0.0\end{array}$ & $\begin{array}{l}1.0 \\
0.3 \\
2.3 \\
1.9 \\
1.5 \\
1.6 \\
2.6\end{array}$ & $\begin{array}{l}\text { V5;C3 } \\
\text { V5;C1;C3 } \\
\text { V5;M1;M3 } \\
\text { V5;M3;C1 } \\
\text { V5;M2;C3 } \\
\text { V5;M3;C3 } \\
\text { V5;M1;M3 }\end{array}$ & $\begin{array}{l}++ \\
\sqrt{ } \\
++ \\
+ \\
++ \\
++ \\
\sqrt{ }\end{array}$ & $\mathrm{TC}_{\mathrm{BOL}-4}=1515.0$ & $\begin{array}{l}1589.24 \\
1528.00 \\
1293.50 \\
1332.50 \\
1435.14 \\
1391.14 \\
1309.50\end{array}$ & $\begin{array}{l}-4.9 \\
-0.9 \\
14.6 \\
12.0 \\
5.3 \\
8.2 \\
13.6\end{array}$ \\
\hline N5 & $\begin{array}{l}-01 \\
-02 \\
-03 \\
-04 \\
-05 \\
-06 \\
-07\end{array}$ & $\begin{array}{l}1494.48 \\
1073.50 \\
938.50 \\
1033.00 \\
958.50 \\
1389.42 \\
1091.00\end{array}$ & $\begin{array}{l}50.6 \\
29.9 \\
61.8 \\
46.4 \\
41.0 \\
73.2 \\
65.6\end{array}$ & $\begin{array}{l}\text { V5;V6;C2;C3 } \\
\text { V5;V6;C1 } \\
\text { V5;V6;M1 } \\
\text { V5;V6;M3;C1 } \\
\text { V5;V6;M2 } \\
\text { V5;V6;M3;C2 } \\
\text { V5;V6;M3;C1 }\end{array}$ & $\begin{array}{l}1133.05 \\
1073.50 \\
938.50 \\
940.50 \\
943.50 \\
1028.14 \\
938.50\end{array}$ & $\begin{array}{l}24.2 \\
0.0 \\
0.0 \\
9.0 \\
1.6 \\
26.0 \\
14.0\end{array}$ & $\begin{array}{l}0.8 \\
1.5 \\
1.7 \\
2.5 \\
2.0 \\
2.8 \\
1.4\end{array}$ & $\begin{array}{l}\text { V5;V6;C3 } \\
\text { V5;V6;C1 } \\
\text { V5;V6;M1 } \\
\text { V5;V6;M2 } \\
\text { V5;V6;M2 } \\
\text { V5;V6;M3;C3 } \\
\text { V5;V6;M1 }\end{array}$ & $\begin{array}{l}++ \\
\sqrt{ } \\
\sqrt{ } \\
++ \\
+ \\
++ \\
++\end{array}$ & $\mathrm{TC}_{\text {BOL-5 }}=1617.0$ & $\begin{array}{l}1333.05 \\
1273.50 \\
1138.50 \\
1140.50 \\
1143.50 \\
1228.14 \\
1138.50\end{array}$ & $\begin{array}{l}17.6 \\
21.2 \\
29.6 \\
29.5 \\
29.3 \\
24.0 \\
29.6\end{array}$ \\
\hline \multicolumn{2}{|c|}{ Mean } & & & & & 3.7 & 1.3 & & & & & 13.6 \\
\hline
\end{tabular}

delivery modes appropriately, performs well in terms of solution quality, and requires an extraordinarily small computation time. Compared to the $\mathrm{OV}^{\mathrm{C}}$ after $50 \mathrm{~h}, \mathrm{RVND}^{\mathrm{SQ}}$ provides significant solution quality improvements for larger problem sets (N4 and N5).

Regarding the economic potentials of the VRPPCdR approach, the mean relative cost improvement of $13.6 \%$ is impressive. Nevertheless, for some instances no cost reduction can be achieved (e.g., N2-01). For these cases, the downsizing of the private vehicle fleets (see Section 5.1) cannot be compensated by the newly available subcontracting options. This fact and the comparison of the used vehicles lead to the conclusion that the available subcontracting possibilities (and their specific parameters) are directly related to the cost reduction potential. In this context, it has to be highlighted that the largest cost reduction for a specific basic data scenario (BOL-1 to BOL-5) is not only achieved with the carrier and rental option scenario S7 (including all possible rental options) but also with carrier and rental option scenarios offering only a subset of all possible rental options (e.g., S4 for BOL-2 or S3 for BOL-4). Because different carrier and rental option scenarios (all with the same parameters) achieve this for different basic data scenarios, we conclude that there is not only one most preferable set of subcontracting options but it depends on basic data scenario.

Summarizing this analysis, it is shown that the VRPPCdR approach leads to notable cost reductions, that the best combination of private fleet and subcontracting options depends on the basic data scenario, and that the available subcontracting possibilities should be considered during fleet size planning to establish a reasonable equipped private fleet.

\section{Conclusions and further research}

In this paper, we tackle a very comprehensive delivery planning problem for shipping companies. It integrates two types of rental options, one based on mileage costs and one based on daily costs, a carrier-dependent cost function considering volumes and distances, and common carriers that offer volume discounts. All these aspects are considered simultaneously 
for the first time and define the new VRPPCdR. The presented MIP specifies the problem in detail and can be used by standard solvers to solve small instances to optimality. Because of the NP-hardness of the VRPPCdR and the goal to solve problem instances of virtually any size, three solution methods based on the principles of VNS are developed: VNS, RVND, and VNSac. The evaluation of these methods shows that RVND enhanced by the explicit shaking mechanism (including the RDtransformation rule) is superior to the other proposed methods. Furthermore, the second enhancement by the distance proportionate vehicle selection mechanism additionally improves the effectiveness of RVND.

The economic potentials of the VRPPCdR approach are assessed based on 35 problem instances and a remarkable mean cost reduction by $13.6 \%$ is reported. Furthermore, we have shown a dependency between the available subcontracting possibilities, basic data scenario, and the economic potentials for cost reduction. In addition, the influence between available subcontracting possibilities on the tactical fleet size planning problem is carved out.

This dependency between the available subcontracting possibilities on the fleet size planning problem suggests further research opportunities. Also, an improvement of the proposed solution methods for very large problem instances, particularly instances with long routes, appears to be a promising issue. For example, ejection chain transformations or the adaptive mechanism proposed by Stenger et al. (2013b) could be integrated. Moreover, the integration of one of the proposed VNSvariants as local search procedure within a population based metaheuristic like a Genetic Algorithm or Ant Colony Optimization seem to be worth to be investigated in future. For example, Nagata and Bräysy (2009) and Böhnlein et al. (2009) have shown the suitability of such approaches to solve VRPs or an VRP variant. In this context, problem specific operators combining solutions with different combinations of used vehicles and common carriers might be interesting.

In addition, the idea of the vehicle selection mechanism for inter-route optimization procedures based on route characteristics (like the absolute distance of their center points) could be extended in the future: For example by considering their relative or absolute position to each other. Also the relative or absolute position of customers could be used to increase the probability of improving $\lambda$-interchanges. Beside the investigation of other solution methods or their components, the problem definition itself could be extended by adding characteristics such as time windows or multiple depots.

\section{Appendix A. Linearization of the last part of objective function (5)}

In a first step, we introduce an auxiliary decision variable $B_{l}$ that indicates whether the minimal transportation rate $t r_{l}^{\text {Min }}$ is used.

$$
B_{l}= \begin{cases}1 & \text { if } \operatorname{tr}_{l}-\left(d f_{l} \cdot \sum_{j \in C^{n}} Z_{j l} \cdot q_{j}\right) \geqslant \operatorname{tr}_{l}^{\text {Min }} \\ 0 & \text { otherwise }\end{cases}
$$

This new variable leads to the following transformation of the last summand of (5):

$$
\begin{aligned}
& \left.\left(\sum_{l \in E} \sum_{i \in C^{n}} Z_{i l} \cdot B_{l} \cdot\left(\operatorname{tr}_{l}-\left(d f_{l} \cdot \sum_{j \in C^{n}} Z_{j l} \cdot q_{j}\right)\right) \cdot \omega_{i l}\right)+\sum_{l \in E} \sum_{i \in C^{n}} Z_{i l} \cdot\left(1-B_{l}\right) \cdot \operatorname{tr}_{l}^{M i n} \cdot \omega_{i l}\right) \\
& \left.\left.=\sum_{l \in E} \sum_{i \in C^{n}} Z_{i l} \cdot B_{l} \cdot \operatorname{tr}_{l} \cdot \omega_{i l}\right)-\left(\sum_{l \in E} \sum_{i \in C^{n}} \sum_{j \in C^{n}} Z_{i l} \cdot B_{l} \cdot d f_{l} \cdot \omega_{i l} \cdot Z_{j l} \cdot q_{j}\right)+\sum_{l \in E} \operatorname{tr} \text { Min } \sum_{i \in C^{n}}\left(Z_{i l}-B_{l} \cdot Z_{i l}\right) \cdot \omega_{i l}\right)
\end{aligned}
$$

However, introducing $B_{l}$ eliminates the maximum operator, but (27) and also (28) remain non-linear. To linearize (28), the product of the decision variables $\left(Z_{i l}, Z_{j l}\right.$, and $\left.B_{l}\right)$ is substituted by two additional auxiliary variables: $P_{i l}$ substitutes the product of $B_{l}$ and $Z_{i l} ; Q_{i j l}$ the product of $B_{l}, Z_{i l}$, and $Z_{j l}$.

$$
\begin{aligned}
& P_{i l}= \begin{cases}1 & \text { if } Z_{i l}=1 \text { and } \mathrm{B}_{l}=1 \\
0 & \text { otherwise }\end{cases} \\
& Q_{i j l}= \begin{cases}1 & \text { if } Z_{j l}=P_{i l}=1, \text { which implicates } Z_{i l}=B_{l}=1 \\
0 & \text { otherwise }\end{cases}
\end{aligned}
$$

After incorporating $P_{i l}$ and $Q_{i j l}$, formula (28) can be written as follows (note that $B_{l}$ is eliminated):

$$
\left.\left.\sum_{l \in E} \operatorname{tr}_{l} \sum_{i \in C^{n}} P_{i l} \cdot \omega_{i l}\right)-\left(\sum_{l \in E} d f_{l} \sum_{i \in C^{n}} \sum_{j \in C^{n}} Q_{i j l} \cdot \omega_{i l} \cdot q_{j}\right)+\sum_{l \in E} \operatorname{tr} r_{l}^{M i n} \sum_{i \in C^{n}}\left(Z_{i l}-P_{i l}\right) \cdot \omega_{i l}\right)
$$

\section{Appendix B. Illustration of the common carriers' cost functions}




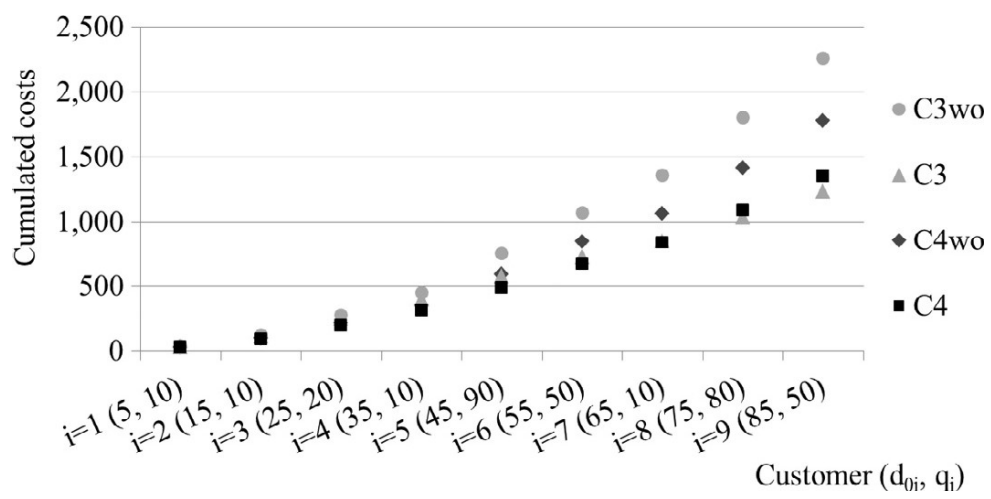

Fig. 2. Cumulated transportation costs with volume discounts (curves C3 and C4) and without volume discounts (curves C3wo and C4wo).

\section{Appendix C. Tables}

Table 14

Reference objective values and relative gaps for instance sets CHU and BOL

\begin{tabular}{lllll}
\hline Instance & Objective value without fixed costs & Relative gap of CPLEX after 50 h [\%] & Fixed costs & Total costs TC \\
\hline CHU-1 & 277.5 & 0.0 & 110 & 387.5 \\
CHU-2 & 366.0 & 0.0 & 220 & 586.0 \\
CHU-3 & 403.5 & 0.0 & 420 & 823.5 \\
CHU-4 & 939.0 & 6.5 & 450 & 1389.0 \\
CHU-5 & 835.5 & 37.3 & 630 & 1465.5 \\
BOL-1 & 313.5 & 0 & 110 & 423.5 \\
BOL-2 & 256.5 & 0 & 220 & 476.5 \\
BOL-3 & 357.0 & 0 & 420 & 777.0 \\
BOL-4 & 1065.0 & 13.1 & 450 & 1515.0 \\
BOL-5 & 987.0 & 27.6 & 630 & 1617.0 \\
\hline
\end{tabular}

Table 15

Basic data with location $(\mathrm{x}, \mathrm{y})$, demand quantities $\left(q_{i}\right)$, and distance to depot $\left(d_{i 0}\right)$.

\begin{tabular}{|c|c|c|c|c|c|c|c|c|c|c|c|c|c|c|}
\hline \multicolumn{5}{|c|}{ Bolduc 1} & \multicolumn{5}{|c|}{ Bolduc 2} & \multicolumn{5}{|c|}{ Bolduc 5} \\
\hline $\mathrm{i}$ & $\mathrm{x}$ & $\mathrm{y}$ & $\mathrm{q}_{\mathrm{i}}$ & $\mathrm{d}_{\mathrm{i} 0}$ & i & $\mathrm{x}$ & $\mathrm{y}$ & $\mathrm{q}_{\mathrm{i}}$ & $\mathrm{d}_{\mathrm{i} 0}$ & i & $\mathrm{x}$ & $\mathrm{y}$ & $\mathrm{q}_{\mathrm{i}}$ & $\mathrm{d}_{\mathrm{i} 0}$ \\
\hline 0 & 35 & 35 & & & 0 & 30 & 40 & & & 0 & 162 & 354 & & \\
\hline 1 & 28 & 59 & 10 & 25 & 1 & 40 & 33 & 7 & 12 & 1 & 111 & 354 & 300 & 51 \\
\hline 2 & 35 & 42 & 7 & 7 & 2 & 26 & 43 & 30 & 5 & 2 & 136 & 355 & 3100 & 26 \\
\hline 3 & 45 & 53 & 13 & 20 & 3 & 47 & 60 & 16 & 26 & 3 & 183 & 401 & 125 & 51 \\
\hline 4 & 54 & 22 & 19 & 23 & 4 & 46 & 26 & 9 & 21 & 4 & 214 & 374 & 100 & 55 \\
\hline \multirow[t]{2}{*}{5} & 68 & 63 & 26 & 43 & 5 & 67 & 25 & 21 & 39 & 5 & 131 & 371 & 200 & 35 \\
\hline & & & & & 6 & 52 & 57 & 15 & 27 & 6 & 215 & 332 & 150 & 57 \\
\hline \multicolumn{5}{|c|}{ Bolduc 4} & 7 & 38 & 31 & 19 & 12 & 7 & 141 & 385 & 150 & 37 \\
\hline i & $\mathrm{x}$ & $\mathrm{y}$ & $\mathrm{q}_{\mathrm{i}}$ & $\mathrm{d}_{\mathrm{iO}}$ & 8 & 36 & 59 & 23 & 19 & 8 & 173 & 334 & 450 & 22 \\
\hline 0 & 266 & 235 & & & 9 & 50 & 39 & 11 & 20 & 9 & 156 & 388 & 300 & 34 \\
\hline 1 & 227 & 276 & 125 & 56 & 10 & 47 & 22 & 5 & 24 & 10 & 164 & 352 & 100 & 2 \\
\hline 2 & 303 & 243 & 84 & 37 & & & & & & 11 & 126 & 341 & 950 & 38 \\
\hline 3 & 312 & 196 & 60 & 60 & \multicolumn{5}{|c|}{ Bolduc 3} & 12 & 104 & 402 & 125 & 75 \\
\hline 4 & 258 & 196 & 500 & 39 & i & $\mathrm{x}$ & $\mathrm{y}$ & $\mathrm{q}_{\mathrm{i}}$ & $\mathrm{d}_{\mathrm{i} 0}$ & 13 & 171 & 346 & 150 & 12 \\
\hline 5 & 286 & 195 & 300 & 44 & 0 & 40 & 40 & & & 14 & 146 & 393 & 150 & 42 \\
\hline 6 & 204 & 186 & 175 & 79 & 1 & 44 & 43 & 18 & 5 & 15 & 201 & 393 & 550 & 55 \\
\hline 7 & 249 & 212 & 350 & 28 & 2 & 42 & 59 & 26 & 19 & 16 & 118 & 354 & 150 & 44 \\
\hline 8 & 209 & 268 & 150 & 65 & 3 & 47 & 57 & 11 & 18 & 17 & 149 & 337 & 100 & 21 \\
\hline 9 & 323 & 212 & 1100 & 61 & 4 & 29 & 55 & 30 & 18 & 18 & 221 & 397 & 150 & 73 \\
\hline 10 & 299 & 267 & 4100 & 45 & 5 & 29 & 30 & 21 & 14 & 19 & 117 & 385 & 400 & 54 \\
\hline 11 & 300 & 254 & 225 & 38 & 6 & 46 & 39 & 19 & 6 & 20 & 138 & 403 & 300 & 54 \\
\hline 12 & 312 & 225 & 300 & 47 & 7 & 67 & 22 & 15 & 32 & 21 & 194 & 409 & 1500 & 63 \\
\hline 13 & 300 & 268 & 250 & 47 & 8 & 64 & 65 & 16 & 34 & 22 & 177 & 361 & 100 & 16 \\
\hline 14 & 305 & 278 & 500 & 58 & 9 & 45 & 52 & 29 & 13 & 23 & 163 & 336 & 300 & 18 \\
\hline 15 & 251 & 238 & 150 & 15 & 10 & 65 & 68 & 26 & 37 & 24 & 158 & 387 & 500 & 33 \\
\hline 16 & 294 & 238 & 100 & 28 & 11 & 33 & 29 & 37 & 13 & 25 & 180 & 392 & 800 & 42 \\
\hline 17 & 256 & 192 & 250 & 44 & 12 & 64 & 24 & 16 & 28 & 26 & 119 & 399 & 300 & 62 \\
\hline 18 & 293 & 202 & 120 & 42 & 13 & 55 & 66 & 12 & 30 & 27 & 128 & 352 & 100 & 34 \\
\hline 19 & 291 & 202 & 600 & 41 & 14 & 44 & 54 & 31 & 14 & 28 & 116 & 346 & 150 & 46 \\
\hline 20 & 221 & 186 & 500 & 66 & 15 & 41 & 22 & 8 & 18 & 29 & 140 & 407 & 1000 & 57 \\
\hline 21 & 317 & 181 & 175 & 74 & & & & & & & & & & \\
\hline 22 & 265 & 206 & 75 & 29 & & & & & & & & & & \\
\hline
\end{tabular}




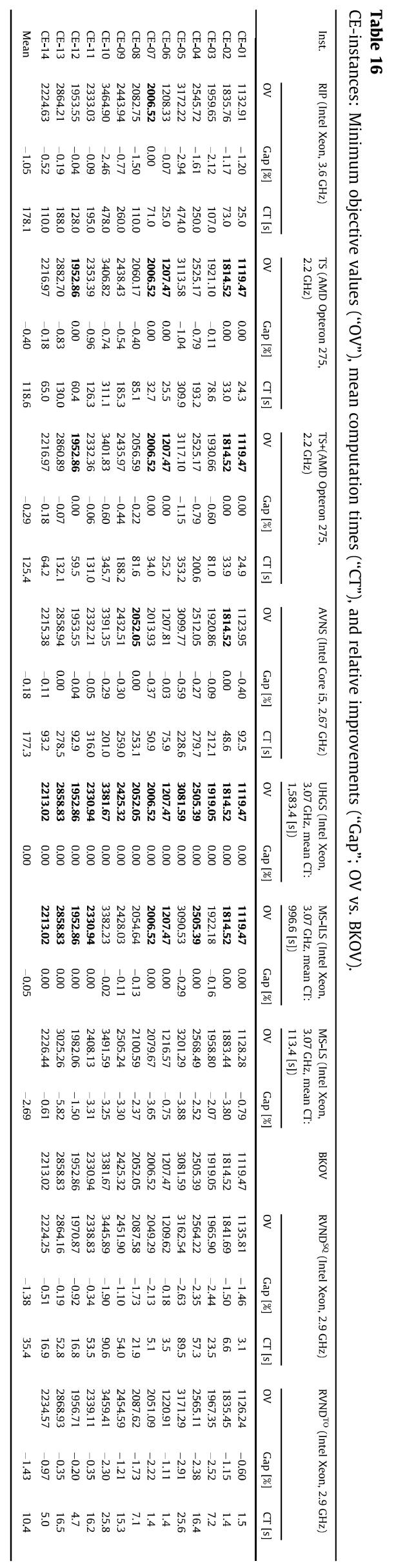




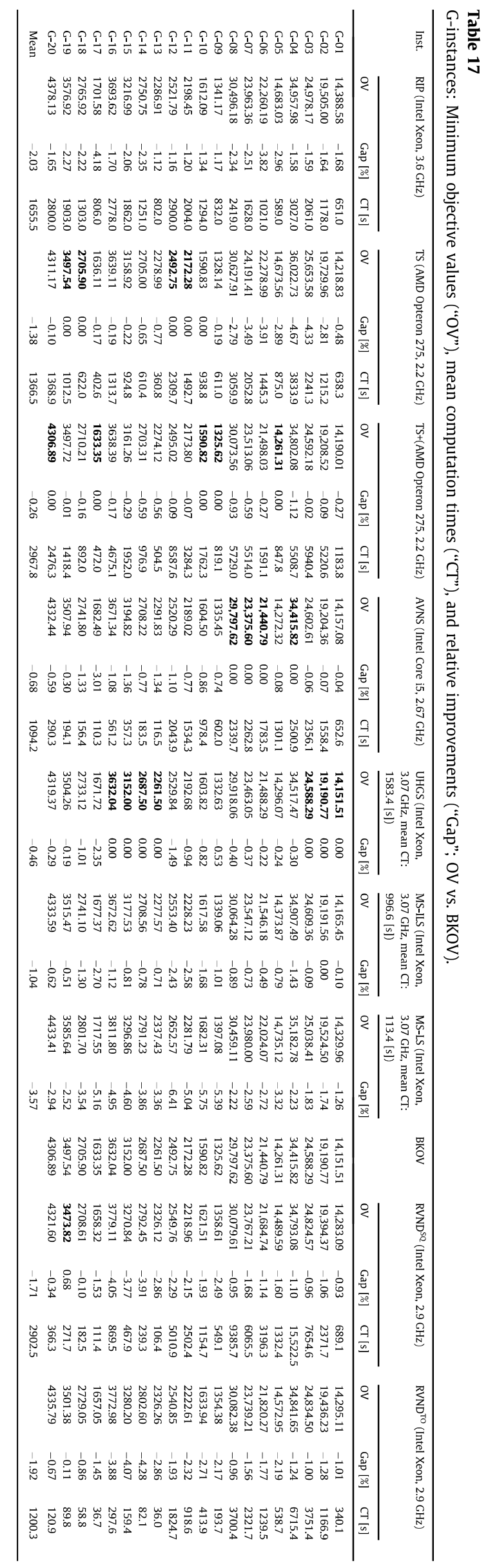




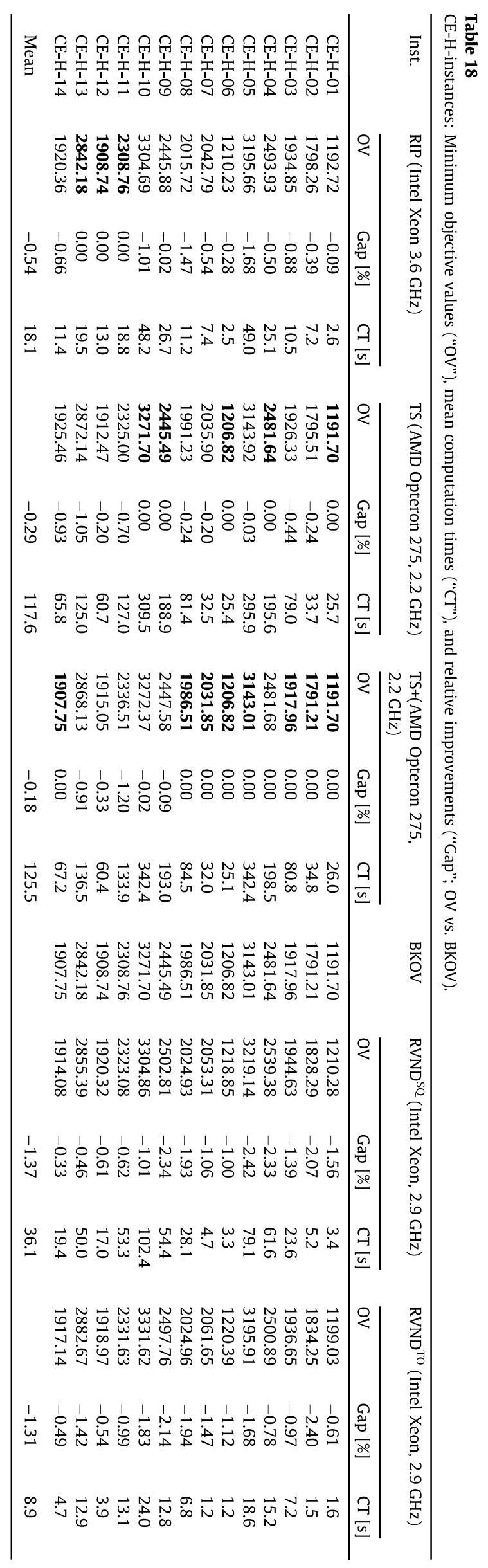




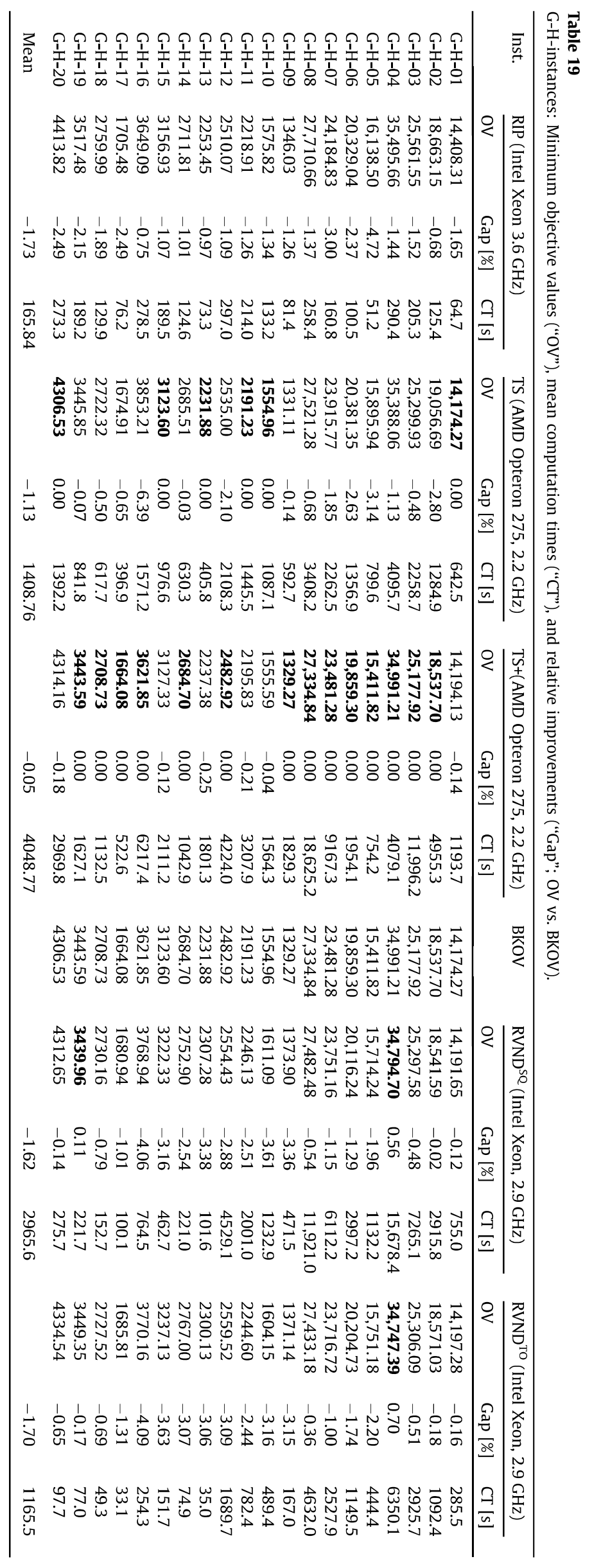




\section{References}

Balas, E., 1989. The prize collecting traveling salesman problem. Networks 19 (6), 621-636.

Balas, E., 2007. The prize collecting traveling salesman problem and its applications. In: Gutin, G., Punnen, A.P. (Eds.), The Traveling Salesman Problem and Its Variations, vol. 12. Springer Science+Business Media, New York, pp. 663-695.

Ball, M.O., Golden, B.L., Assad, A.A., Bodin, L.D., 1983. Planning for truck fleet size in the presence of a common-carrier option. Decis. Sci. 14 (1), 103-120. Bienstock, D., Goemans, M., Simchi-Levi, D., Williamson, D., 1993. A note on the prize collecting traveling salesman problem. Math. Program. 59 (1-3), 413420.

Böhnlein, D., Gahm, C., Tuma, A., 2009. A hybrid meta-heuristic for the VRPTW with cluster-dependent tour starts in the newspaper industry. In: IEEE Computer Society (Ed.), The 42nd Annual Hawaii International Conference on System Sciences (HICSS-42 2009). 5-8 January 2009, Waikoloa, Big Island, HI, USA, pp. 1-10.

Bolduc, M.-C., Renaud, J., Boctor, F.F., 2007. A heuristic for the routing and carrier selection problem. Eur. J. Oper. Res. 183 (2), $926-932$.

Bolduc, M.-C., Renaud, J., Boctor, F.F., Laporte, G., 2008. A perturbation metaheuristics for the vehicle routing problem with private fleet and common carriers. J. Oper. Res. Soc. 59 (6), 776-787.

Bräysy, O., 2003. A reactive variable neighborhood search for the vehicle-routing problem with time windows. INFORMS J. Comput. 15 (4), $347-368$.

Campbell, J.F., 1990. Freight consolidation and routing with transportation economies of scale. Transp. Res. Part B: Methodol. 24 (5), $345-361$.

Christofides, N., Eilon, S., 1969. An algorithm for the vehicle-dispatching problem. Oper. Res. 20 (3), 309.

Chu, C.-W., 2005. A heuristic algorithm for the truckload and less-than-truckload problem. Eur. J. Oper. Res. 165 (3), $657-667$.

Clarke, G., Wright, J.W., 1964. Scheduling of vehicles from a central depot to a number of delivery points. Oper. Res. 12 (4), $568-581$.

Côté, J.-F., Potvin, J.-Y., 2009. A tabu search heuristic for the vehicle routing problem with private fleet and common carrier. Eur. J. Oper. Res. 198 (2), 464469.

Dantzig, G.B., Fulkerson, R., Johnson, S., 1954. Solution of a large-scale travelling salesman problem. Inst. Oper. Res. Manage. Sci. 2 (4), $393-410$.

Defryn, C., Sörensen, K., Cornelissens, T., 2016. The selective vehicle routing problem in a collaborative environment. Eur. J. Oper. Res. 250 (2), 400-411.

Diaby, M., Ramesh, R., 1995. The distribution problem with carrier service: a dual based penalty approach. ORSA J. Comput. 7 (1), $24-35$.

Gahm, C., Dünnwald, B., Sahamie, R., 2014. A multi-criteria master production scheduling approach for special purpose machinery. Int. J. Prod. Econ. 149, 89-101.

Golden, B.L., Wasil, E.A., Kelly, J.P., Chao, I.-M., 1998. The impact of metaheuristics on solving the vehicle routing problem: algorithms, problem sets, and computational results. In: Crainic, T.G., Laporte, G. (Eds.), Fleet Management and Logistics. Springer Science+Business Media, New York, pp. 33-56.

Grefenstette, J.J., 2000. Proportional selection and sampling algorithms. In: Bäck, T., Fogel, D.B., Michalewicz, Z. (Eds.), Evolutionary Computation. Taylor \& Francis, New York, London, pp. 172-180.

Hall, R.W., Racer, M., 1995. Transportation with common carrier and private fleets: system assignment and shipment frequency optimization. IIE Trans. 27 (2), 217-225.

Hansen, P., Mladenović, N., 2001. Variable neighborhood search: principles and applications. Eur. J. Oper. Res. 130 (3), $449-467$.

Hansen, P., Mladenović, N., Moreno Perez, J.A., 2010. Variable neighbourhood search: methods and applications. Ann. Oper. Res. 175 (1), $367-407$.

Helsgaun, K., 2000. An effective implementation of the Lin-Kernighan traveling salesman heuristic. Eur. J. Oper. Res. 126 (1), $106-130$.

Helsgaun, K., 2009. General K-opt submoves for the Lin-Kernighan TSP heuristic. Math. Program. Comput. 1 (2-3), 119-163.

Hemmelmayr, V.C., Doerner, K.F., Hartl, R.F., 2009. A variable neighborhood search heuristic for periodic routing problems. Eur. J. Oper. Res. 195 (3), 791802.

Hertz, A., Mittaz, M., 2001. A variable neighborhood descent algorithm for the undirected capacitated arc routing problem. Transp. Sci. 35 (4), $425-434$.

Johnson, D.S., McGeoch, L.A., 2007. Experimental analysis of heuristics for the STSP. In: Gutin, G., Punnen, A.P. (Eds.), The Traveling Salesman Problem and Its Variations, vol. 12. Springer Science+Business Media, New York, pp. 369-443.

Karapetyan, D., Gutin, G., 2011. Lin-Kernighan heuristic adaptations for the generalized traveling salesman problem. Eur. J. Oper. Res. 208 (3), $221-232$.

Klincewicz, J.G., Luss, H., Pilcher, M.G., 1990. Fleet size planning when outside carrier services are available. Transp. Sci. 24 (3), $169-182$.

Kopfer, H., Wang, X., 2009. Combining vehicle routing with forwarding - extension of the vehicle routing problem by different types of sub-contraction. J. Kor. Inst. Ind. Eng. 35 (1), 1-14.

Krajewska, M.A., Kopfer, H., 2009. Transportation planning in freight forwarding companies: tabu search algorithm for the integrated operational transportation planning problem. Eur. J. Oper. Res. 197 (2), 741-751.

Kytöjoki, J., Nuortio, T., Bräysy, O., Gendreau, M., 2007. An efficient variable neighborhood search heuristic for very large scale vehicle routing problems. Comput. Oper. Res. 34 (9), 2743-2757.

Lenstra, J.K., Rinnooy Kan, A.H.G., 1981. Complexity of vehicle routing and scheduling problems. Networks 11 (2), $221-227$.

Lin, S., 1965. Computer solutions of the traveling salesman problem. Bell Syst. Tech. J. 44 (10), 2245-2269.

Lin, S., Kernighan, B.W., 1973. An effective heuristic algorithm for the traveling-salesman problem. Oper. Res. 21 (2), 498-516.

Liu, R., Jiang, Z., Liu, X., Chen, F., 2010. Task selection and routing problems in collaborative truckload transportation. Transp. Res. Part E: Logist. Transp. Rev. 46 (6), 1071-1085.

Miller, C.E., Tucker, A.W., Zemlin, R.A., 1960. Integer programming formulations and traveling salesman problems. J. Assoc. Comput. Mach., 326-329

Mladenović, N., Hansen, P., 1997. Variable neighborhood search. Comput. Oper. Res. 24 (11), 1097-1100.

Nagata, Y., Bräysy, O., 2009. Edge assembly-based memetic algorithm for the capacitated vehicle routing problem. Networks 54 (4), $205-215$.

Nguyen, H.N., Rainwater, C.E., Mason, S.J., Pohl, E.A., 2014. Quantity discount with freight consolidation. Transp. Res. Part E: Logist. Transp. Rev. 66, 66-82.

Osman, I.H., 1993. Metastrategy simulated annealing and tabu search algorithms for the vehicle routing problem. Ann. Oper. Res. 41 (4), $421-451$.

Özkaya, E., Keskinocak, P., Roshan Joseph, V., Weight, R., 2010. Estimating and benchmarking Less-than-Truckload market rates. Transp. Res. Part E: Logist. Transp. Rev. 46 (5), 667-682.

Polacek, M., Hartl, R.F., Doerner, K.F., 2004. A variable neighborhood search for the multi depot vehicle routing problem with time windows. J. Heuristics 10 (6), 613-627.

Potvin, J.-Y., Naud, M.-A., 2011. Tabu search with ejection chains for the vehicle routing problem with private fleet and common carrier. J. Oper. Res. Soc. 62 (2), 326-336.

Rieksts, B.Q., Ventura, J.A., 2008. Optimal inventory policies with two modes of freight transportation. Eur. J. Oper. Res. 186 (2), $576-585$.

Sheen, G.-J., Tsao, Y.-C., 2007. Channel coordination, trade credit and quantity discounts for freight cost. Transp. Res. Part E: Logist. Transp. Rev. 43 (2), 112128.

Stenger, A., Schneider, M., Goeke, D., 2013a. The prize-collecting vehicle routing problem with single and multiple depots and non-linear cost. EURO J. Transp. Logist. 2 (1-2), 57-87.

Stenger, A., Vigo, D., Enz, S., Schwind, M., 2013b. An adaptive variable neighborhood search algorithm for a vehicle routing problem arising in small package shipping. Transp. Sci. 47 (1), 64-84.

Sun, L., Karwan, M.H., Gemici-Ozkan, B., Pinto, J.M., 2015. Estimating the long-term cost to serve new customers in joint distribution. Comput. Ind. Eng. 80, $1-11$.

Tersine, R.J., Barman, S., 1991. Economic inventory/transport lot, sizing with quantity and freight rate discounts. Decis. Sci. 22 (5), $1171-1179$.

Toptal, A., Bingöl, S.O., 2011. Transportation pricing of a truckload carrier. Eur. J. Oper. Res. 214 (3), 559-567.

Tsao, Y.-C., Lu, J.-C., 2012. A supply chain network design considering transportation cost discounts. Transp. Res. Part E: Logist. Transp. Rev. 48 (2), $401-414$.

Turkensteen, M., Klose, A., 2012. Demand dispersion and logistics costs in one-to-many distribution systems. Eur. J. Oper. Res. 223 (2), $499-507$. 
Vahrenkamp, R., 2011. The Calculation of Costs for Delivery Routes (No. Report 71/2011. Kassel. Retrieved from <http://www.vahrenkamp.org/Nr71_Cost_ Delivery.pdf>.

Vidal, T., Maculan, N., Ochi, L.S., Vaz Penna, P.H., 2015. Large neighborhoods with implicit customer selection for vehicle routing problems with profits. Transp. Sci. Articles Adv., 1-15

Volgenant, T., Jonker, R., 1987. On some generalizations of the travelling-salesman problem. J. Oper. Res. Soc. 38 (11), 1073-1079.

Wang, X., Kopfer, H., 2014. Collaborative transportation planning of less-than-truckload freight. OR Spectrum 36 (2), $357-380$.

Wang, X., Kopfer, H., Gendreau, M., 2014. Operational transportation planning of freight forwarding companies in horizontal coalitions. Eur. J. Oper. Res. 237, 1133-1141.

Weng, K.Z., 1995. Modeling quantity discounts under general price-sensitive demand functions: optimal policies and relationships. Eur. J. Oper. Res. 86 (2) 300-314.

Wilcox, J.B., Howell, R.D., Kuzdrall, P., Britney, R., 1987. Price quantity discounts: some implications for buyers and sellers. J. Market. 51 (3), 60.

Yaman, H., 2006. Formulations and valid inequalities for the heterogeneous vehicle routing problem. Math. Program. 106 (2), 365-390.

Ziebuhr, M., Kopfer, H., 2014. The integrated operational transportation planning problem with compulsory requests. In: González-Ramírez, R.G., Schulte, F. Voß, S., Ceroni Díaz, J.A. (Eds.), Lecture Notes in Computer Science, Computational Logistics. Valparaiso, Chile. Springer International Publishing, Cham, Heidelberg, NewYork, Dordrecht, London, pp. 1-15. 